\title{
Acute Bulbäraffection (Embolie der Art. cerebellar. post. inf. sinistr. ?).
}

Von

\author{
Dr. Adolf Wallenberg
}

in Danzig.

\begin{abstract}
Die Mittheilung eines Falles von acut entstandener Läsion der Medulla oblongata bedarf eigentlich einer Rechtfertigung, wenn die Beobachtung bisher lediglich eine klinische sein konnte und vorläufig auch bleiben wird. Das einheitliche Krankheitsbild indessen, welches sich unschwer aus einer Menge auffallender Symptome construiren lässt, wird der im Folgenden mitgetheilten Krankengeschichte ein gewisses Interesse wohl auch dann noch sichern, wenn die für meine Diagnose angeführten Gründe sich später als unzureichend erweisen sollten.

Der Kranke ist ein 38jähriger Seilermeister. Sein Vater starb angeblich infolge einer Hirnblutung. Die Mutter litt seit ihrem 17. Lebensjahre an Schwäche auf beiden Augen, wurde mehrmals operirt, ist aber seit vielen Jahren röllig erblindet. Von seinen Geschwistern starb eine Schwester im 12. Jahre an Unterleibsentzündung, ein Bruder wurde während seiner Militärzeit aus Versehen erschossen. - Unser Patient, jetzt die einzige Stütze seiner blinden Mutter, kam - so giebt er an — schon mit linksseitigem Staar zur Welt, auch auf dem rechten Auge konnte er seit der frühesten Jugend nicht ordentlich sehen. Als Knabe wurde er einmal in Danzig, einmal in Königsberg an den Augen operirt. Seitdem konnte er in der Nähe grössere Gegenstände erkennen, mit Hilfe einer blauen Convexbrille grobe Druckschrift lesen, anch ziemlich leserlich schreiben. Der genauere Befund an den Augen folgt weiter unten, ich erwähne an dieser Stelle nur, dass derselbe Status schon vor $4 \frac{1}{2}$ Jahren von mir aufgenommen worden ist, dass also von frischen Störungen nicht die Rede sein kann. Bei der Anfertigung und dem Verkaufe von Seilerwaaren war er infolge seines geschwächten Sehvermögens hauptsächlich auf
\end{abstract}


einen hoch ausgebildeten Tastsinn angewiesen. Seiner Angabe nach ist er geschlechtlich nie inficirt gewesen, Potus und starker Tabakgenuss wird zugegehen. Ich behandelte den Patienten zuerst im Jahre 1889 an einer Perityphlitis, wurde in den folgenden Jahren wiedorholt von ihm consultirt, weil sich Erscheinungen bei ihm einstellten, die auf eine Fettdegeneration des Herzmuskels hinwiesen (Athemnoth, starke Vermehrung der Pulsfrequenz, Verbreiterung der Herzdämpfung). Zu diesen Beschwerden gesellten sich im Anfange des Jahres 1892 häufige Anfälle von Blutandrang nach dem Kopfe, Schwindel, Herzklopfen; darauf folgte wieder eine längere Pause, in welcher er sich eines relativen Wohlseins erfreute.

Am Abend des 9. September 1893 kam er von einem Begräbniss, fühlte sich schon auf dem Heimwege etwas unwohl, fröstelte und hatte die Absicht, früh ins Bett zu gehen. Zu Hause angelangt, wurde er von einem Schwindelanfalle niedergeworfen, zugleich stellten sich sehr heftige Schmerzen im linken Ange ein, welche sich bald auf die ganze linke Gesichtshälfte verbreiteten. Das Bewusstsein verlor er nicht für einen Augenblick. Ausser dem Schwindel und Gesichtsschmerz, dessen Intensität bis zum anderen Morgen nicht wesentlich abnahm, bemerkte der Kranke noch, dass er heiser war und nicht schlucken konnte, wie er meinte, infolge starker Schleimanhäufung im Rachen.

\section{Status am 10. September 1893.}

Kräftig gebauter Mann. Starker Panniculus adiposus auf Brust und Bauch. Alte kleine Narben in der rechten Achselhöhle und am rechten Unterschenkel. Patient nimmt die passive Rückenlage ein. Beim Versuche sich aufzurichten stellt sich starker Schwindel ein, und er fällt mit ziemlich grosser Vehemenz nach links hinüber. Sensorium vollständig klar. Sprache ohne deutliche Störung (ausser der Heiserkeit). Die Untersuchung der Hirnnerren ergiebt:

Geruch: beiderseits erhalten.

A ugen: Kleine Bulbi. Rechte Cornea in der Mitte weisslich verfärbt und vorgewölbt; Iris an dieser Narbe adhärent. Die Pupille hat nach oben innen einen Ausschnitt (artificielles Colobom). Medien klar. Untersuchung des. Augenhintergrundes sehr erschwert. Papille erscheint im umgekehrten Bilde als grosse ron oben nach unten gerichtete Ellipse. Temporalwärts von ihr grauweisse halbkreisförmige Verfärbung. Linke Cornea durchsichtig, vordere Kammer sehr tief. Pupille (mit Ausnahme eines nach oben gerichteten artificiellen Coloboms) durch eine weissliche Membran verschlossen, an welche die Iris excentrisch nach oben angeheftet ist. Papille elliptisch (grosse Achse von oben nach unten). Prüfung auf Reaction der Pupillen wegen der Synechieen unmöglich. Sehschärfe beiderseits stark herabgesetzt, rechts mehr wie links (Finger werden in ca. 1 Heter Entfernung erkannt).

Augenmuskeln: Geringer Strabismus convergens. Nystagmus horizontalis und verticalis (auch beim Fixiren clonische Zuckungen nach rechts und links).

Trigeminus: Bei Berührung der linken Cornea und Conjunctiva erfolgt 
kein Lidschluss, während die betr. Reflexe rechts prompt auszulösen sind. Im übrigen besteht in der ganzen linken Gesichtshälfte ausser spontanem Schmerz eine deutliche Hyperaesthesie für Berührung (Stecknadelkopf stets als Spitze gefühlt). Dieselbe erstreckt sich nicht auf die Schleimhaut des Mundes. In der rechten Gesichtshälfte dagegen werden Stiche als blosse Berührung gefühlt, kalt wird für warm gehalten, leise Berührungen orkennt Patient oft gar nicht.

Kaubewegungen werden beiderseits mit gleicher Kraft ausgeführt.

Geschmack an vorderer und hinterer Zungenhälfte völlig normal.

Facialis: In der Stellung der Lider, der Nasolabialfalten, der Mundwinkel keine Differenz, weder in der Ruhe noch bei Bewegungen.

Gehör: Normale Hörweite auf beiden Ohren.

Glossopharyngeus - Vagus - Aceessorius bulbaris: Schlucken sehr erschwert; feste Speisen können gar nicht, flüssige nur tropfenweise mit grosser Anstrengung geschluckt werden. Rechte Gaumensegelhälfte steht etwas tiefer als die linke (wohl infolge starker Vergrösserung der rechten Tonsille); beide Seiten heben sich beim Anlauten sehr wenig. Bei Berührung des weichen Gaumens und Rachens wird kein Schlingreflex ausgelöst. In Folge dessen gelingt die laryngoskopische Untersuchung trotz des schweren Krankheitszustandes überraschend leicht. Dabei zeigt sich eine vollständige Lähmung des linken Stimmbandes: dasselbe bewegt sich weder bei Respiration noch bei Phonation. Die bestehende Heiserkeit ist wohl auf diese Paralyse zurückzuführen.

Hypoglossus: Die Zunge wird gerade herausgestreckt, nach allen Seiten hin gut bewegt; bei ruhiger Lage im Munde ist die linke Hälfte des Zungengrundes stärker gewölbt als die rechte.

Rumpf und Extremitäten: Auf der ganzen rechten Körperhälfte totaler Verlust des Schmerz- und Kältegefühls. Auf der linken Seite geringe Hyperaesthesie. Patient klagt über Schwäche im linken Arme, bei genacerer Prüfung ist aber weder rechts noch links eine Störung der groben Kraft zu constatiren. Auch in der Action der Sternocleidomastoidei und Cucullares keine Differenz zu Ungunsten einer Seite. Dagegen besteht eine deutliche Ataxie der linken Extremitäten. Der Kranke greift mit der rechten Hand richtig ans linke Ohr und an die Nase, fährt aber mit der linken Hand vorbei. Ebenso legt er die rechte Hacke sicher aufs linke Knie, die linke indess gewöhnlich weit oberhalb der rechten Kniescheibe, dabei unsicheres Herumfahien.

Reflexe: Plantarreflexe normal, Cremasterreflex fehlt links. Kniereflex beiderseits nicht auszulösen.

Brustorgane: Lungen ohne nachweisbare Anomalie. Herzchoc schwach, diffus, im 5. Intercostalraume; relative Dämpfung rechts bis zum rechten Sternalrande, links fast $3 \mathrm{Ctm}$. über die linke Mammillarlinie; Herztöne dumpf, kein Geräusch. Puls 76-82 pro Minute, weich; keine Differenz zwischen rechter und linker Radialis; Arterienrohr nicht rigide (auch Temporales nicht geschlängelt).

Bauchorgane: Leber ragt um ca. 2 Ctm. unter dem rechten Rippen. 
bogen hervor (in der rechten Mammillarlinie). Milz nicht nachweisbar vergrössert. Urin ohne Eiweiss and Zucker.

Temperatur normal.

11. September: Schwindelgefühl etwas geringer. Auch der Schmerz im linken Trigeminusgebiete hat nachgelassen. Cornealreflex links erloschen, rechts erhalten. Hyperaesthesie der rechten Gesichtshälfte nahezu verschwunden, an den rechten Extremitäten und der rechten Rumpfhälfte dagegen besteht die Analgesie und Thermanaesthesie noch fort. Ataxie der linken Extremitäten sehr ausgesprochen, Hyperaesthesie der linken Körperhälfte kaum mehr nachzuweisen. Plantarreflex beiderseits erhalten. Cremasterreflex fehlt links. Bauchreflexe beiderseits verschwunden, ebenso die Patellarreflexe. Lähmung des linken Stimmbandes und Schlinglähmung unverändert. Auf der linken Seite wird vollständige Analgesie der Nasenschleimhant und des Nasenflügels constatirt. Auch im Bereiche des harten und weichen Gaumens scheint links die Schmerzempfindung herabgesetzt zu sein.

12. September: Das Schwindelgefühl geringer (bei ruhiger Lage). Patient giebt an, beim Oeffnen der Augen die Wände von rechts oben nach links unten schräg stehend zu sehen. Keine Doppelbilder.

Sensibilität: Gefühl von Pelzigsein in der linken Gesichts- und Stirnhälfte. Keine Schmerzen im Gebiete des linken Trigeminus. Corneal- und Conjunctivalreflex links völlig erloschen, rechts erhalten.

Tastgefühl überall im Wesentlichen normal (leichte Pinselberührung wird wahrgenommen and richtig localisirt). Schmerzgefühl stark herabgesetzt in der linken Gesichts- und Stirnhälfte, der rechten Kinngegend (nach oben bis zur Linie Mund - rechtes Ohr, weiter oben normal), der rechten Körperhälfte vom Kinn abwärts.

Temperaturgefühl: Kälte (Eis) wird in der eben bezeichneten Zone (linke Stirn, Nase, Wange, rechte Körperhälfte vom Kinn abwärts) als warm gefühlt, Temperaturdifferenzen bis ca. $45^{\circ} \mathrm{C}$. nicht erkannt. Die Anaesthesie schneidet genau in der Mittellinie ab, links von derselben am Halse, Rumpf, Arm und Bein normale, sogar sehr difficile Unterscheidung geringster Differenzen.

Münzen werden mit beiden Händen gleich richtig erkannt. Die Schleimhaut des Mundes und Rachens zeigt keine gröberen Sensibilitätsstörungen.

Notilität: Keine Differenz in der Stirn- und Gesichtsmusculatur beider Seiten. Stirnrunzeln, Augenschluss, Naserümpfen, Lachen, Pfeifen wird beiderseits gleich gut ausgeführt. Heiserkeit, Zungendifferenz, Schluckbeschwerden unverändert. Grobe Kraft der Extremitäten bei einfachen und Widerstandsbewegungen rechts und links gleich gut. Ataxie der linken Extremitäten sehr ausgesprochen (statt eines Kreises wird ein Polygon resp. Zickzack mit dem linken Beine beschrieben ete. siehe oben), Patellarreflex links nicht auszulösen, rechts ist er wiedergekehrt; es lässt sich der Cremasterreflex beiderseits hervorrufen, links jedoch schwerer als rechts. Bauchreflexe fehlen.

13. September: Patellarreflex links auch durch Jendrassik nicht auszulösen, rechts erhalten. Plantar- und Cremasterreflex beiderseits nor- 
mal. Links im Stirn-Augen-Nasengebiet Analgesie und Thermanaesthesie, dagegen in linker Wangen-Ohr-Kinn-Gegend nur noch unerhebliche Abschwächung aller Gefühlsqualitäten. In der Motilität und Sensibilität der Extremitäten und des Rumpfes keine Aenderung. Pulsfrequenz: 104-112.

15. September: Cornealreflex links erloschen. Gefühl für grobe Temperaturdifferenzen und stärkere Nadelstiche ist in der linken Stirn und Wange zurückgekehrt, ebenso in den rechten Fingerspitzen. Die übrigen Partieen zeigen noch dieselben Störungen wie vorher. Schwindel, Heiserkeit, Hypoglossusparese unverändert. Schmerzen im Hinterlopf, Schlaflosigkeit. Puls: 82.

17. September: Herpesbläschen, in rundlichen Gruppen angeordnet, an der linken Nasenhälfte, dem linken Mundwinkel und der linken Oberlippenhälfte, in Form schmaler Ellipsen an der Regio deltoidea und inguinalis dextra. Von der linken Cornea und Conjunctiva aus kein Lidreflex anszulösen, dagegen erfolgt bei Berührung der Cilien prompter Schluss der Lider. Während die Störung des Temperatursinns dieselben Regionen einnimmt wie früher, beginnt die Herabsetzung des Schmerzgefühls erst unterhalb einer Linie vom Ansatz des zweiten Rippenknorpels zum Tubercul. majus des rechten Humerus. An den Fingerspitzen völlig normale Sensibilität. Hautreflexe wie oben. Patellarreflex links wiedergekehrt, doch schwächer als rechts. Ataxie hat bedeutend. abgenommen, namentlich am linken Arme, lässt sich aber noch deutlich (einigen Collegen) demonstriren. Recurrenslähmung und Zungendifferenz unverändert. Flüssige Speisen lrönnen schon besser geschluckt werden. Schlaf besser. Puls: 100 .

In den nächsten Tagen traten starke Schmerzen im rechten Auge auf, die Narbe auf der Cornea röthete sich, dabei starke Ciliarinjection; auch im Nacken heftige Schmerzen, Pulsfrequenz ging wieder bis unter 90 pro Minute herunter. Ob ein Druck auf das rechte Ange bei der Untersuchung oder die Verabreichung von Jodkali als Ursache dieser Reizerscheinungen aufzufassen ist, wage ich nicht zu entscheiden. Nach Ansetzen von 5 Blutegeln an die Schläfen verschwanden die Schmerzen sowohl wie Entzündungserscheinungen sehr bald.

Der Herpes blieb bis zum 22. September sichtbar.

24. September: Wieder anfallsweise auftretende Schmerzen, jetzt im Nacken und in der linken Schläfengegend. Puls geht bis auf 84 pro Minute herunter. Dabei Taubheitsgefühl der linken Stirn- und Schläfenregion. Linker Cornealreflex erloschen; dabei wird jede Berührung der Cornea und Conjunctiva deutlich wahrgenommen. Die Sensibilitätsstörung der linken Gesichtshälfte am stärksten ausgeprägt in der unmittelbaren Umgebung des Auges, am linken Nasenrücken und besonders in der Schleimhaut der linken Nasenhöhle. Am linken harten, weniger am weichen Gaumen scheint eine geringere Empfindlichkeit für Nadelstiche zu bestehen (unsichere Angaben). In der rechten Hohlhand, an den Fingern, der rechten Fusssohle und den Zehen ziemlich normale Sensibilität. An den übrigen Regionen der rechten Körperhälfte dieselben Störungen wie oben.

Rechte Gaumenhälfte steht zwar etwas tiefer: als die linke, contrahirt 
sich aber bei der Phonation besser. Zunge im Munde links voluminöser als rechts. Linkes Stimmband steht bei Phonation und Respiration still. Bewegungen der Extremitäten geschehen im Bett beiderseits mit gleicher Kraft. Ataxie des linken Armes undeutlich, am linken Beine sehx ausgesprochen. Patellarreflex beiderseits gleich; kein Fussclonus. Radialis-, Biceps-, Tricepsreflex beiderseits undeatlich.

Erster Gehversuch: Beim Aufstehen starkes Schwanken nach links. Gehen nur mit Unterstützung beider Arme möglich. Dabei schleppt Patient das linke Bein zuerst nach (Andeutung von Spasmus), dann wirft er es gewaltsam, fast schleudernd vor. Nach drei Schritten Neigung auf die linke Seite zu fallen.

26. September: Zweiter Gehversuch gelingt besser. Schwindel nur bei geöffneten Augen; bei geschlossenen steht Patient gerade, hat aber beim Oeffnen der Augen sofort Neigung nach links zu fallen. Schwächegefühl im linken Beine. Zuweilen Schmerzen and Paraesthesieen längs des linken Extensor und Abductor pollicis longus, ausserdem im linken Talocruralgelenk. Geschmack auf beiden Zungenhälften gleich gut, Puls: 96.

Urin 1020 sp. Gew., ohne Eiweiss und Zucker, wirkt stark reducirend.

30. September: Corneal- und Conjunctivalreflex links erloschen. Lidschluss bei Berührung der Cilien. Gaumenbogen steht links etwas höher als rechts, hebt sich aber beim Anlauten rechts viel mehr als links, die Uvula wird dabei nach rechts hin verzogen, und es kommt eine deutliche Einkerbung rechts von derselben zu Stande. Gesichtsmuskeln beiderseits gleich gut contrahirt.

Kehlk op : Bei Inspiration geringe Auswärtsbewegung des linken Stimmbandes, bei Intonation dagegen völliger Stillstand, dabei geht das rechte Stimmband über die Mittellinie hinüber.

Sensibilität des Larynx anscheinend normal (Sonde wird rechts und links gleich gut gefühlt). Beim Gehen weniger Schwindel, besonders wenn Patient mit geschlossenen Augen geht. Linkes Bein wird deutlich schleudernd vorgesetzt; kein Spasmus.

2. October. Patient geht ohne Unterstützung zwei Schritte, dabei schloppt er das linke Bein nicht nach, schleudert es ein wenig; taumelt auch bei offenen Augen nicht mehr, wenn er mit geschlossenen Füssen steht. Beim Gehen stellt sich aber bald Schwindel ein. Taubheitsgefühl in der linken Gesichtshälfte und den rechten Extremitäten. An den linken Augenlidern und den angrenzenden Hautpartieen (besonders Stirn, Nasenrücken, innerer Lidwinkel) werden Tasteindrücke gut localisirt, die Unterscheidung von Spitze und Knopf (bei leiser Berührung); sowie von warm und kalt ist weit unsicherer als rechts. An der Schleimhaut der linken Nasenhöhle wird auch bei tiefem Stich mit der Spitze der Nadel nur Knopf gefühlt. Abwärts von einer Linie Mund - linkes Ohrläppchen ist die Sensibilität vollständig normal. Schmerz- und Temperaturgefühl im rechten Oberarm, rechter Ellenbeuge, recbtem Oberschen. kel sehr stark herabgesetzt, am Unterarm, Handrücken, Unterschenkel, Fuss: rücken verringert (erst bei tieferen Stichen und grösseren Temperaturdifferenzen 
richtige Angaben), Hohlhand und Fusssohle ohne stärkere Anomalie. Am Rumpfe reicht die Abschwächung des Kältegefühls bis zum rechten Unterkieferrande hinauf, für die Störung des Schmerzgefühls bildet die Linie 2. Rippenknorpel-rechtes Tubercul. majus humeri die obere Grenze. Puls: 96.

4.-16. October. Allmälig bessert sich der Gang des Patienten, Schwindel und Neigung nach links (zuweilen auch nach rechts) zu fallen vermindert sich. Die Ataxie im linken Beine ist noch deutlich, im Arme dagegen nahezu verschwunden. Beim Schlucken fester Speisen noch starke Beschwerden, Flüssigkeiten werden gut geschluckt. Linkes Stimmband wie früher. Zunge gerade herausgestreckt, im Munde links voluminöser. Gaumensegel wie oben. Patient schreibt und liest mit blauer Convexbrille wie vor seiner Erkrankung. Die Besserung der Sensibilitätsstörungen macht Fortschritte am rechten Unterarme, rechter Hand and rechtem Oberschenkel, am rechten Unterschenkel und Fusse fast normale Verhältnisse. Am meisten beeinträchtigt sind jetzt (ausser der linken Gesichtshälfte) rechte Rumpfhälfte (Rücken, Brust und Bauch, für Temperatur auch Hals) und reehter Oberarm.

Drucksinn: Am linken Arme wird bei 1.00 Gramm Belasting Zu- resp. Abnahme von 15 Gramm gefühlt, am rechten bei gleicher Belastung erst 50 Gramm Differenz.

Kraftsinn: Mit den Händen können beim Heben von 250 Gramm beiderseits 20 Gramm Zunahme resp. Abnahme gefühlt werden.

(Diese Prüfungen wurden schon am 4. October angestellt. Später ausgeführte genauere Untersuchungen ergaben etwas andere Resultate, siehe unten).

18. October: Bei längerem Gehen tritt Schwanken nach links auf. Patient giebt an, nicht unterscheiden zu können, ob dabei Schwindel eintritt, oder ob das linke Bein plötzlich seinen Dienst versagt. Die linke Unterextremität schlendert noch etwas beim Gehen. Mit dem linken Fusse wird noch statt eines Kreises ein Polygon beschrieben, die linke Ferse wird unsicher auf das rechte Knie gelegt; auch die Lage des linken Beines beurtheilt Patient schechter als die des rechten. Im linken Arme keine deutliche Ataxie. Patellarreflexe beiderseits gleich. Radialreflex links zwar schwächer, aber deutlich, Anconaeusreflex beiderseits schwach. Patient klagt über wechselnde Schmerzen in der linken Regio supraorbitalis (keine Druckpunkte) and im Nacken. Puls 90.

26. October: Grosse Unsicherheit im linken Beine beim Herabsteigen einer Treppe, das Hinaufsteigen ging besser.

\section{Prüfung der faradocutanen Sensibilität (mit Knopfelektrode):}

Rollenabstand in $\mathrm{Mm}$.

Rollenabstand in Mm.

Erstes Auftreten einer Empfindung Erstes Auftreten von Schmerz rechts links rechts links

$\begin{array}{lllrr}\text { Stirn . . . . . . . . . } & 130 & 105 & 100 & 90 \\ \text { Wange. . . . . . . . } 120 & 105 & 90 & 85 \\ \text { Schulter . . . . . . } & 120 & 115 & 65 & 80\end{array}$


Rollenabstand in $\mathrm{Mm}$. Rollenabsfand in $\mathrm{Mm}$. Erstes Auftreten einer Empfindung Erstes Auftreten von Schmerz

\begin{tabular}{|c|c|c|c|c|}
\hline & rechts & links & rechts & links \\
\hline Oberarm & 105 & 105 & 60 & 75 \\
\hline Enterarm & - 115 & 125 & 70 & $70-75$ \\
\hline Handrücken & 95 & 95 & 60 & 70 \\
\hline Brust . . . & . 115 & 125 & 75 & 95 \\
\hline Bauch & - 120 & 115 & 70 & 90 \\
\hline Oberschenkel. & 110 & 105 & 70 & 80 \\
\hline Unterschenkel & 100 & 110 & 60 & 70 \\
\hline Fussrücken & 80 & 80 & 55 & 70 \\
\hline Rücken . . & 105 & 105 & 55 & 85 \\
\hline
\end{tabular}

Bei Wiederholungen dieser Prüfung schwankte das Ergehniss um 5 bis $20 \mathrm{Mm}$., doch blieb das Verhältniss der einzelnen Regionen zu einander nahezu constant.

27. October. Prüfung mit 'Tasterzirkel: (Mittel aus mehreren Untersuchungen.)

Abstand der Zirkelenden, welcher zur Erzeugung einer Doppelempfindung nothwendig war, in Millimetern.

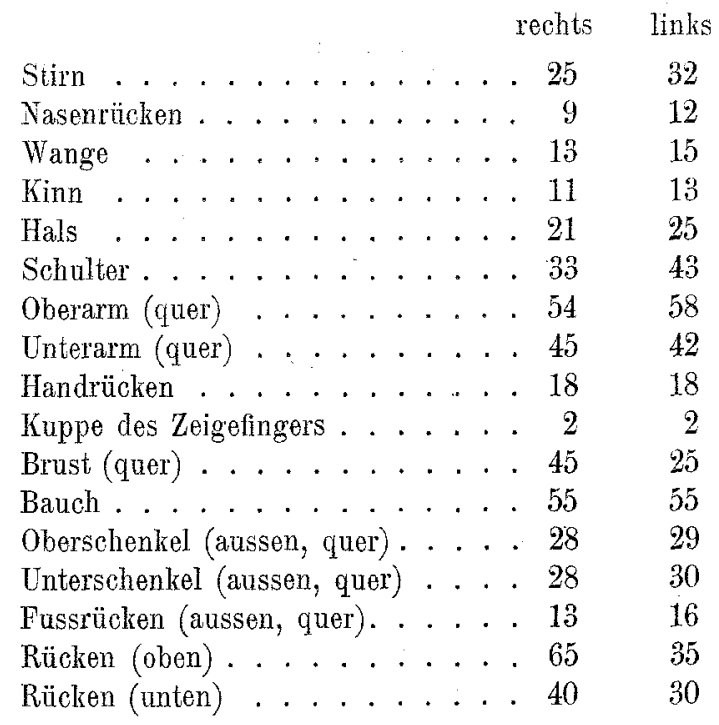

29. October bis 6. November: Geringe Besserung der Sensibilitätsanomalien. Linkes Stimmband bewegt sich auch beim Anlauten etwas, bleibt indessen gegenüber dem rechten weit zurück. Ataxie des linken Beines kaum noch angedeutet. Hautreflexe mit Ausnahme des fehlenden epigastrischen Reflexes beiderseits gleich gut auszulösen. Sehnenreflexe kommen rechts und links mit gleicher Intensität zu Stande. Zunge und Gaumensegel wie oben. 
9. November: Vorstellung des Patienten in einer Sitznng des Danziger ärtlichen Vereines. Es konnten folgende Symptome einem grösseren Zuhörerkreise demonstrirt werden.

1. Schwanken nach der linken Seite beim Gehen.

2. Geringe Andeutung einer Ataxie des linken Beines.

3. Parese des linken Stimmbandes (Herr Sanitätsrath Dr. 'T'ornwaldt fïhrte in dankenswerther Weise die Untersuch mo aus und theilte das Resultat den Anwesenden mit).

4. Zungendifferenz (grösseres Volumen der linken Zungenhälfte bei ruhiger Lage im Munde).

5. Aufhebung (bei Druck mit dem Nadelkopfe gegen die Cornea resp. Conjunctiva nur sehr erhebliche Abschwächong) des Corneal- und Conjunctivalreflexes der linken Seite.

6. Störung des Schmerz- und Temperatursinns im Bereiche der linken Stirn, der linken Augenlider, des linken Nasenrückens mit den angrenzenden Wangenpartieen, der linken Nasenschleimhaut, der rechten Rumpfhälfte und der rechten Extremitäten.

15. November-2. December: Genauere Prüfung der Schmerz- und Temperaturempfindung ergiebt folgende Ausdehnung der Störung (siehe die Abbildungen). Die doppelt schraffirten Stellen bezeichnen jene Regionen, an welchen tiefere Nadelstiche noch als Kopf gefühlt, Temperaturdifferenzen von $30-40^{\circ}$ C. nicht erkannt werden. An den einfach aber eng schraflirten Partieen können Spitze und Kopf bei festem Andrücken, Temperaturunterschiede yon $20-30^{\circ} \mathrm{C}$. wahrgenommen weroen. An den weiter schrafiliten Stellen ondlich sind die Sensibilitätsanomalien nahezu verschwunden (es bestehen jedoch immerhin noch deutliche Unterschiede gegenüber gleichen Stellen der anderen Seito).

In dieser Zeit litt Patient wieder häufiger an Schmerzen im linken Auge und im Nacken, klagte über Anfälle ron Beängstigungen. Die Herzdämpfung reicht noch über den rechten Sternalrand hinaus. Puls 96-100.

5. December: Linkes Stimmband bleibt bei der Phonation gegen das rechte zurẗck. Es ist schmäler und nicht so glänzend weiss gefärbt wie das rechte.

12. December: Prüfung des Druckgelühls:

Bei 75 Gramm Belastung werden unterschieden (in Theilen der Belastung ausgedrückt): rechts

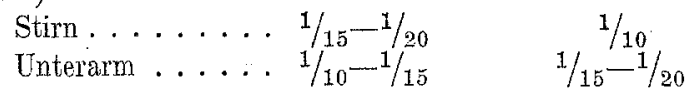

Prüfung des Kraftsinns:

Bei 90 Gramm Belastung werden durch Aufheben eines mit der Hand gefassten und mit den Gewichten armirten Tuches unterschieden:

$\begin{array}{cc}\text { rechts } & \text { links } \\ 1 / 18-1 / 20 & 1 / 12\end{array}$




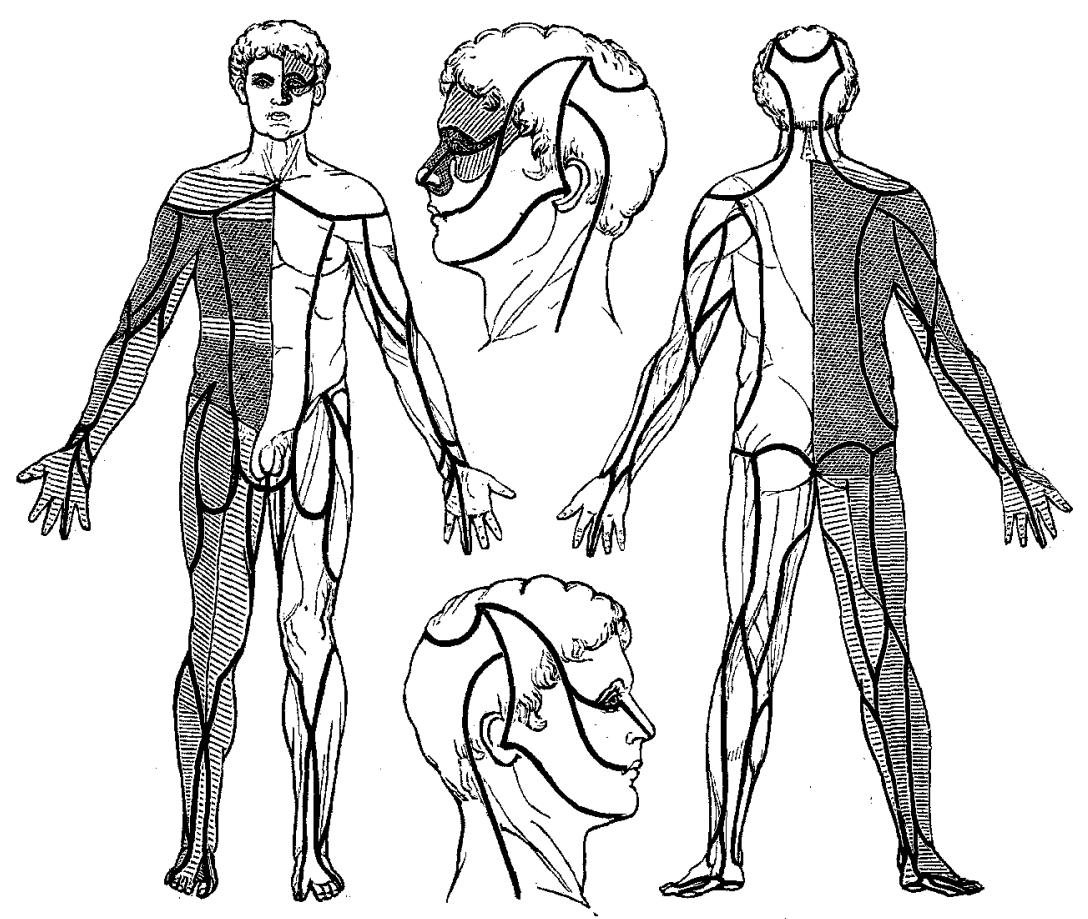

Schmerzemp findung resp. Unterscheidung von Spitze und Kopf einer Stecknadel. In Bezug auf die Bedeutung der verschiedenen Schraffirung siehe Text.

15. December: Otitis externa sinistra.

22. December: Zunge im Munde links mehr gewölbt, kann gat bewegt werden. Medianfurche geradlinig. Gaumen hebt sich beim Anlauten besses rechts, Uvula nach rechts gekrümmt, beim Phoniren bildet sich derContractionswinkel rechts von der Uyula.

29. December: Patient klagt über Sausen in beiden Ohren, besonders im rechten; er giebt an, gleiche Beschwerden zeitweise schon in früheren Jahren gehabt zu haben, aber nicht so heftig wie jetzt. Ob früher beide Ohren gleichmässiger rom Sausen befallen waren, weiss er nicht bestimmt. Bei Auscultation des linken Warzenfortsatzes und der angrenzenden Theile der Scheitel- resp. Hinterhauptsregion nur Athmungsgeräusch. Zwischen dem rechten Processus mastoideus dagegen und der hinteren Medianlinie lautes systolisches Geräusch, das sich nach unten bis zur Theilungsstelle der Carotis communis dextra fortpflanzt, nach vorne bis zur rechten Schläfe (sehr schwach!) hörbar ist. Seine grösste Intensität erreicht es dicht oberhalb der (stärker als links pulsirenden) Theilungsstelle der Carotis communis.

12. Januar 1894. Herzdämpfung rechts $1 \mathrm{Ctm}$. über rechten Sternalrand, links $4 \mathrm{Ctm}$. über linke Mammillarlinie hinausreichend, geht nach oben 


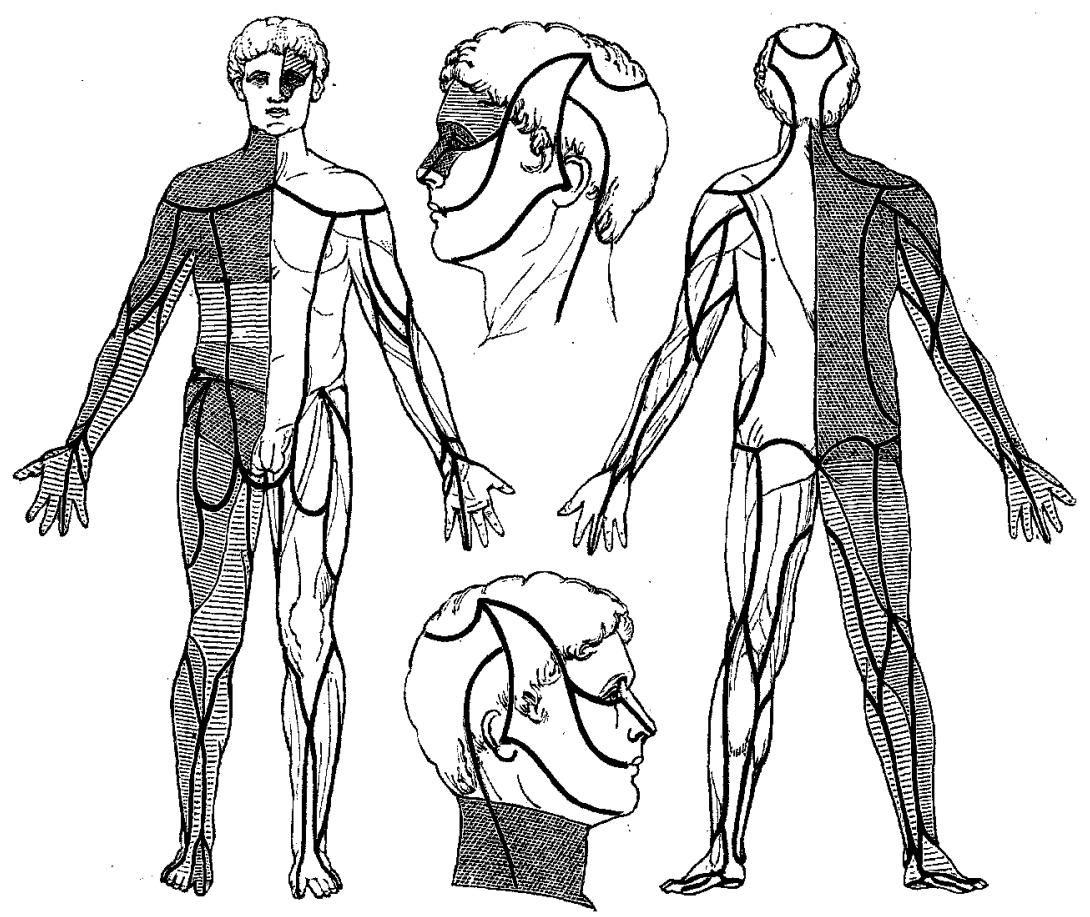

Temperaturempfindung. In Bezug auf die Bedentung der verschiedenen Schraffirung siehe Text.

bis zum Unterrande der dritten Rippe. Herztöne rein. Puls 96. Rechte Carotis communis pulsirt stärker als die linke, auch die Anonyma nimmt an der Pulsation theil. Auf der rechten Carotis communis zwei reine Töne. Keine Differenz der Blutwelle an den Radiales. Das Geräusch am Proc. mastoid. dext. und der Carotis interna, noch in gleicher Weise hörbar, verschwindet nicht bei vorsichtiger Compression der Carotis communis dicht unterhalb der Theilungsstelle.

16. Februar: Die Beschwerden des Patienten haben sich gebessert, öfters stellen sich aber Beklemmungen und Augenflimmern ein. Puls 96. Befund am Herzen, den grossen Gefässen und am Proc. mastoideus dext. derselbe wie oben. Nach Compression der Carotis communis wird das Geräusch noch wahrgenommen. Zungengrund steht links höher als rechts. Gaumen hebt sich rechts besser als links, Uvula wird bei der Phonation mit den benachbarten Theilen des linken Gaumenbogens nach rechts hinüber gezogen.

Sensibilität des weichen Gaumens nicht merklich vermindert (auch kalt and warm gut unterschieden). Keine Schluckbeschwerden; aber Patient klagt über Schleimanhäufung im Rachen. Linkes Stimmband wieder deutlicher 
paretisch (Patient hat gesungen), das rechte geht bei der Phonation über die Mittellinie hinüber. Die Temperaturempfindung hat am Nasenrücken sich etwas gebessert, an den übrigen Theilen der linken Gesichts- und rechten Körperhälfte ist sie noch unverändert, soweit ich prüfen konnte. Subjective Kältecmpfindung an der rechten Hand; Patient giebt an, schon bei geringer Abkühlung eine Verminderung seiner Schmerz- und Temperaturempfindung an der rechten Hand und den Fingern zu merken. Neigung, nach links zu fallen, ist nicht mehr nachzuweisen.

15. März. Wieder stärkere Beklemmungen. Befund am Herzen unverändert. Erster Ton an der rechten Carotis communis unrein, geht nach oben in das beschriebene systolische Geräusch über, dessen Intensität iusofern wechselt, als es bald unterhalb des Processus mastoideus lauter ist, bald auf dem Warzenfortsatze selber.

Linkes Stimmband nahezu völlig paralytisch (steht bei Phonation und Respiration still, ist kürzer und schmäler als das rechte, welches bei der Phonation die Mittellinie iberschreitet, und concav*).

Bevor ich in eine kurze Recapitulation der Daten eingehe, welche uns die vorstehende Krankengeschichte an die Hand giebt, glaube jch darauf hinweisen zu müssen, dass die Untersuchung im Hause des $\mathrm{Pa}$ tienten resp. in meiner Sprechstube stattgefunden hat, in Folge dessen vielleicht nicht immer mit der Präcision vorgenommen werden konnte, wie sie in eịner Klinik möglich ist. Folgendes kann ich wohl als Resultat meiner Beobachtung hinstellen:

Ein 38 jähriger Mann, durch Sehschwäche infolge congenitaler Augenaffection (linksseitige operirte Cataract, Cornealnarbe und vordere Synechie rechts, Capsel- resp. Exsudatreste und Anheftung der Iris an dieselben links, beiderseits starke Verzerrung der Pupille, Coloboma artificiale, Astigmatismus, Nystagmus horizontalis und verticalis) zur feineren Ausbildung seines Tastsinns gezwungen, seit Jahren an Fettdegeneration des Herzens leidend (wahrscheinlich Folge chronischer Alcohol- und Nicotinintoxication), bekommt nach kurzem Unwohlsein einen Schwindelanfall ohne Bewusstseinsverlust, zugleich Schmerzen und Hyperästhesie der linken, Hypästhesie der rechten Gesichtshälfte, Verlust der Schmerzund Kälteempfindung in den rechten Extremitäten und der rechten

*) Anmerkung bei der Correctur: Im September 1894, Januar und März 1895 wioderholte Untersuchungen ergaben, dass die oben beschriebenen Störungen der Stimmband-, Gaumen-, Zungenbewegung einerseits, der Schmerzund Temperaturempfindung andererseits, allerdings in erheblich geringerem Grade, noch fortbestehen. Auch das Geräusch am rechten Warzenfortsatz ist unrerändert. 
Rumpfhälfte bei anscheinend vollständiger Erhaltung der Berührungsempfindung, Schlinglähmung, Sensibilitätsstörung der Mund-, Rachen-, Gaumenschleimhaut, Bewegungsstörung des weichen Gaumens, am ersten Tage beiderseits, später links, ohne Störung in der Innervation der Gesichtsmuskeln, totale linksseitige Recurrenslähmung, Parese des linken Musc. hyoglossus (so dente ich nach Gowers") die Differenz in dem Volumen der Zunge bei ruhiger Lage im Munde), Ataxie der linken Extremitäten ohne nachweisbare Störung der groben Kraft, Neigung nach links zu fallen, Fehlen beider Patellarreflexe, Bauchreflexe und des linken Cremasterreflexes, endlich relative Pulsverlangsamung gegen früher (von 96 bis auf $76-82$ ).

In den nächsten Tagen wird die Sensibilität der rechten Gesichtshälfte wieder normal, die Hyperästhesie der linken Körperhälfte (welche ich oben zu erwähnen versänmt habe) verschwindet, die des linken Trigeminusgebietes verwandelt sich in eine Anaesthesie vorzugsweise für Schmerz- und Kältempfindung (weniger für Ortssinn und electrocutane Sensibilität) mit Aufhebung des Corneal- resp. Conjunctivalreflexes. Der Patellarreflex der rechten Seite kehrt sehr bald zurück, fehlt aber links während der nä.chsten 7 Tage, der Puls beschleunigt sich wieder, die übrigen Störungen bleiben. Am achten Tage erscheint ein Herpes entsprechend den analgetischen Zonen: linkes Gesicht (mit Nasenschleimhaut, während Mund-Rachensensibilität wiedergekehrt ist), rechteSchulter, rechte Weiche, der Patellarreflex tritt auch links wieder auf. $Z_{w e i}$ bis drei Monate nach dem Insult etwa folgender Status:

a) Subjective Symptome:

1. Schwindel, Neigung nach links zu fallen.

2. Taubheitsgefühl in der linken Gesichtshälfte, rechten Körperhalfte mit Ausnahme des Gesichtes.

3. Schluckbeschwerden (sehr gering).

4. Schmerzen im Nacken, zuweilen auch im linken Auge.

b) Objective Symptome:

1. Schwanken nach links beim Gehen.

2. Ataxie der linken Extremitäten (nur noch angedeutet).

3. Parese der linken Gaumensegelhälfte.

4. Paralyse, später Parese des linken Stimmbandes (Andentung von Atrophie?).

5. Grösseres Volumen der linken Zungenhälfte bei ruhiger Lage im Munde.

*) Gowers, Handbuch der-Nervenkrankheiten. Deutsche Ausgabe von Dr. Karl Grube. 1892. II. Bd. S. 292. 
6. Sensibilitätsstörung im Bereiche des ersten, weniger des zweiten Astes des linken Trigeminus, namentlich Auge, Lider, Nasenrücken und Nasenschleimhaut betreffend (die Ausdehnung entspricht ungefähr dem Verlaufe des Nervus nasociliaris und einiger Aeste des Lacrymalis sinistr.), in geringerem Grade Stirn- und Jochbogenregion. Die Anomalien betreffen zwar hauptsächlich Schmerz- und Temperatursinn, doch ist auch (siehe Tabellen) die electrocutane Sensibilität, Ortssinn und Drucksinn in geringem Maasse betheiligt.

7. Fehlen des linken Corneal- und Conjunctivalreflexes.

8. Störungen des Schmerz- und Kältegefühls*) an der rechten Rumpfhälfte (Temperatursinn bis zum rechten Unterkieferrande aufgehoben, Schmerzgefühl dagegen nur bis zu einer Linie vom Ansatz der zweiten Rippe bis zum Tubercul. maj. humer.). Der Grad dieser Störung ist am stärksten auf dem Rücken, Brust oberhalb der Mammilla, Bauch unterhalb des Nabels, Schulter, Aussenseite des Oberarms, dann folgt die Aussenseite des Unterarms, des Beines, gleich stark ist die Anomalie im Bereiche des Endastes vom Nerv. peroneus profundus dext., nahezu normal wird die Sensibilität an den Innenseiten beider Extremitäten, Hand, Fuss, oberer Bauchregion.

9. Alterationen der übrigen Empfindungsqualitäten (namentlich Ortssinn, faradocutane Sensibilität, Drucksinn) in geringem Grade. Wichtig erscheint mir einmal die geringe Grösse der Tastkreise, wohl eine Folge der feineren Ausbildung des Tastsinns; zweitens die Localisation der merklichen Differenzen: an Rücken und Brust rechts, Schulter und Hals links grössere Durchmesser der Tastkreise. An der Grenze von Hals und Schulter konnte man mit dem Inductionsapparat noch starke Unterschiede zu Ungunsten der rechten Seite wahrnehmen, während Nadelspitze und Kopf gleich deutlich auf beiden Seiten erkannt wurde. Der geringen Einbusse, welche der Drucksinn auf der rechten, der Kraftsinn auf der linken Seite erlitten hat, lege ich kein besonderes Gewicht bei, erwähne sie aber hier, weil ich später noch darauf zurückkommen werde.

*) Charakteristisch war und ist zum Theil anch noch die Angabe des P., bei niederen Temperaturen (anch Eis) nur ein Wärmegefühl zu empfinden. Man könnte daraus auf eine isolirte Affection der Fasern für die Kälteempfindung schliessen. Wenn aber eine solche auch überwiegt, so haben jedenfalls auch die anderen Elemente für Temperaturempfindungen gelitten, denn bei Berührung rerschieden stark erhitzter Gegenstände wurde die Differenz nicht erkannt. 
10. Andentung von Ataxie des linken Beines ohne Störung der groben Kraft.

11. Fehlen der Banchreflexe.

In den folgenden Wochen verschwinden allmälig die Schluckbeschwerden, die Neigung nach links zu fallen, die Ataxie; die anderen Erscheinungen, namentlich die Stimmband-, Zungen-, Gaumenparese und die Sensibilitätsstörungen bleiben im Wesentlichen stationär. Dazu gesellt sich ein neues Symptom:

12. Bei der Auscultation des rechten Warzenfortsatzes systolisches Geräusch, das sich nach hinten bis zur Crista occipitalis, nach unten bis zum Unterkieferwinkel, nach vorne (sehr schwach!) bis zur Schläfe verfolgen lässt. Pulsation der Halsgefässe rechts stärker als links.

Das Geräusch ist wahrscheinlich schon vor langer Zeit hörbar gewesen, gelangte aber erst dann zur Perception, als der Patient über Sausen in den Ohren, namentlich im rechten, klagte. Durch Compression der rechten Carotis communis dicht unterhalb der Theilungsstelle kann es nicht ganz unterdrückt werden.

Nach dieser summarischen Beschreibung der Störungen wird es unsere Aufgabe sein, erstens den Sitz der Affection zu ermitteln, zweitens, soweit es möglich ist, die Frage zu beantworten: Welcher pathologische Process liegt hier vor? - Die Localdiagnose ist meiner Meinung nach in unserem Falle nicht allzu schwer, wenn wir sorgfältig abwägen, welche Symptome noch als Insulterscheinungen aufzufassen sind, welche Störungen als passagere und bleibende Herdsymptome gelten müssen. Berücksichtigen wir ausserdem die Resultate der pathologischen und experimentellen Forschung, so kann der Ausbau der Diagnose auf einem relativ gesicherten Fundamente unternommen werden. Ich will dabei gleich bemerken, dass die congenitale resp. früh erworbene Anomalie der Augen des Patienten zwar eine Prüfung des Pupillarreflexes verbinderte, dass aber eine irgend erhebliche Aenderung des übrigen Befundes an den Augen als Folge des Insults ausgeschlossen werden kann; andererseits erleichterte die hohe Ausbildung der Hautsensibilität ungemein die Untersuchung der betreffenden Störungen, namentlich beim Vergleich mit der gesunden Seite. Da eine Trübung des Bewusstseins bei dem Einsetzen des Insults nicht stattgefunden hat, werden wir diejenigen Symptome als Insulterscheinungen ansehen müssen, welche schon in den nächsten Tagen wieder verschwanden. Als solche begegnen uns: 1. eine relative Pulsverlangsamung*), 2. eine Hypaesthesie für

*) Später auftretende geringere Bradycardieen zeigten sich stets in Verbindung mit Nackenschmerzen etc., dürften also rorübergehenden Gehiruhyperämien zuzuschreiben sein. 
Schmerz- and Temperaturempfindung in der rechten Gesichtshälfte, 3. eine geringe Hyperaesthesie der linken Körperhälfte, 4. vorübergehende Aufhebung des Sehnenreflexes am rechten Knie (für zwei Tage, während der linke Patellarreflex erst am achten Tage wieder hervorgerufen werden konnte und auch dann weit schwächer als der rechte ausfiel). Auf das Verhalten der Hautreflexe gehe ich hier nicht weiter ein. Wenn diese rasch vorübergehenden Erscheinungen für die Herdbestimmung auch nebensächlich sind, so werden wir doch später auf dieselben zurücklommen, sobald es sich um eine genauere Bestimmung: des Krankheitsprocesses handelt.

Schwieriger ist schon die Trenung der passageren von den bleibenden Herdsymptomen, da eine continuirliche Besserung fast aller Störungen beobachtet werden konnte. Zur Beantwortung der Frage, an welcher Stelle wir den Krankheitsherd zu erwarten haben, genügt es, vorläufig diese Sonderung nicht vorzunehmen. Lässt uns doch die Stimmbandlähmung, die Zungen- und Gaumenparese der linken Seite mit boher Wahrscheinlichkeit eine Affection der Medulla oblongata, und zwar ihrer linken Hälfte, vermutben. Genauer bestimmen wir die Höhe dadurch, dass wir untersuchen, welche Hirnnerven ergriffen, welche freigeblieben sind.

Gehen wir vom Cervicalmark aufwärts, so kann eine Betheiligung des spinalen Accessorius ausgeschlossen werden (Gleichstand der Schultern, beiderseits gleich gute Action des Sternocleidomastoideus und Trapezius); der Hyoglossus kann nur in einzelnen Wurzelfädchen (resp. Kernzellen) getroffen sein, da die Beweglichkeit der Zunge vollständig frei ist, also nur der Tonus des Hyoglossus eine Einbusse erlitten hat, aber diese Parese ist eine dauernde. Der linke Accessorius muss, soweit er dem verlängerten Marke entstammt, in allen seinen Aesten stark afficirt sein (Stimmbandlähmung, Gaumensegelparese). In wie weit der Vagus und Glossopharyngeus an der Affection des Gaumens und an der Schlinglähmung betheiligt ist, lässt sich schwer entscheiden, weil die anatomischen und physiologischen Untersuchungen über die Innervation des Pharynx und der Gaumenmusculatur noch nicht abgeschlossen sind*). Da jedoch keine andauernde Sensibilitätsstörung des weichen Gaumens, keine Alteration des Geschmacks bestand, so werden wir nicht fehl gehen, wenn wir die oberste Grenze der Affection nicht über die untersten Glossopharyngeuswurzeln hinausverlegen. Das Gebiet des Acusticus

*) Siehe Réthi, Der periphere Lauf der motorischen Rachen- und Gaumennerven (Sitzungsber. der Kais. Academie der Wissenschaften. Mathematisch-naturwissenschaftliche Klasse. Bd. CII. Abth. III. März 1893). 
und Facialis fällt nicht mehr in den Bereich des Herdes (man könnte versucht sein, die Gaumensegelparese auf eine Facialisstörung zu beziehen; nachdem jedoch wiederholt:) eine Gaumensegelparese bei isolirter Accessoriuserkrankung und ein Freibleiben des Gaumens bei einer Läsion des Facialis centralwärts yom Knie beobacbtet worden ist, balte ich eine Heranziehung dieses Nerven in unserem Falle nicht nur für überflüssig, sondern für direct fehlerhaft). Auch die Augenmuskelnerven dürften von diesem Process kaum tangirt worden sein (Nystagmus und Strabismus schon vorher constatirt, keine Doppelbilder; die Angabe des Kranken in den ersten Tagen, dass er die Wände schräge stehen sieht, findet ihre Erklärung in den gleich zu erwähnenden Gleichgewichtsstörungen). Opticus und Olfactorius endlich sind ganz unbetheiligt. -Wir können demnach annehmen, dass durch die läsion die linke Hälfte der Medulla oblongata etwa von der Beendigung der Pyramidenkrenzung bis zum Austritt oberer Vagus- resp. unterer Glossopharyngeuswurzeln betroffen ist, und dass die Intensität der anatomischen Veränderung von unten nach oben hin abnimmt. Den linken Trigeminus habe ich bisher absichtlich unerwähnt gelassen, weil wir seine Störung nicht nur für die Höhen- sondern auch für die Querschnittsbestimmung benutzen können. - Legen wir an der Stelle einen Frontalschnitt durch den Bulbus, wo die Pyramidenkreuzung eben beendet ist (siehe oben) und prüfen zugleich, ob Erscheinungen vorhanden sind, welche auf eine Destruction der Theile hinweisen, denen wir in diesem Schnittbilde begegnen. Fangen wir von der ventralen Fläche an: Die Pyramidenfasern müssen im Wesentlichen intact sein, denn alle Bewegungen geschehen von Anfang an mit normaler Kraft und Extensität (eventuell könnte die Ataxie der linken Extremitäten auf eine Läsion noch ungekreuzter Pyramidenfasern der linken Seite zurückgeführt werden). Die lateral von den Pyramiden austretenden Hypoglossuswurzeln sind (entweder nach ihrem Durchtritt oder intrabulbär) in geringer Anzahl und in geringem Grade afficirt. Dorsalwärts von diesen treffen wir die inneren Nebenoliven resp. den Anfang der Oliven. Wir können über deren Function, also auch über eine Störung derselben, nichts Sicheres aussagen, wenden uns daher weiter nach hinten zu den hier austretenden Fasern des Accessorius und seinem motorischen Kern. Beide fallen in den Bereich des Herdes (Stimmbandlähmung, event. Gaumensegelparese). Der Austrittsstelle des Accessorius sehr nahe liegt die Kleinhirnseitenstrangbahn im Begriff dorsalwärts zu ziehen. Eine Trennung derselben in die vor*

*) Réthi und Gowers a. a. O. S. 232 und 233. 
dere und hintere Portion ${ }^{*}$ ) hätte für nnseren Zweck keinen grossen Werth, ich kann mich also darauf beschränken, nach Symptomen zu suchen, welche anf eine (dauernde oder vorübergehende) Unterbrechung: derjenigen Leitungsbahnen hinweisen, die Rückenmarkszellen mit Kleinhirnwurm verbinden. Dass diese Fasern Beziehungen zum Muskelsinn, spec. zur Coordination der Bewegungen derselben Seite besitzen, wird heute wohl kaum mehr bestritten; fraglicher ist ihr Einfluss auf das Zustandekommen der Sehnenrefiexe. Dass ein solcher besteht, möchte ich auf Grund der Beobachtungen annehmen, in denen bei Kleinhirnaffectionen die Patellarreflexe fehlten ${ }^{*}$ ). Wir werden also die in unserem Falle so evident hervortretende Ataxie der linken Extremitäten mit einiger Wahrscheinlichkeit, das anfängliche Fehlen des linken Patellarreflexes dagegen (anch die Störung des Kraftsinns im Bereiche des linken Armes?) nur mit grosser Reserve auf eine Affection der linken Kleinhirnseitenstrangbahn zurückführen, welche sich allmälig wieder ausgeglichen hat ${ }^{* * *}$ ). Neben der Ataxie und Reflexanomalie haben wir bei unserem Patienten eine auffällige Neigung, nach links zu fallen, constatiren können - ein Symptom, das zwei Monate nach dem Insult noch in einer Sitzung des Danziger ärztlichen Vereins hat demonstrirt werden können. Diese Erscheinung kann, meiner Meinung nach, lediglich durch eine Betheiligung des linken Corpus restiforme und des Kleinhirnwurmes erklärt werden, denn sie ist eine exquisit cerebellare Störung und vollkommen unabhlängig von der Ataxie, welche einen rein spinalen Charakter trug. Demnach sind wir zu der Annahme gezwnngen, dass sich die Läsion an der dorsolateralen Fläche des Bulbus weiter proximalwärts erstreckt als an der ventralen, ja wahrscheinlich das Kleinhirn noch mit in ihren Bereich gezogen hat. Die Gangstörung ist

*) Loewenthal (Rev. medic. de la Suisse Romande 1885), Tooth (Gulstonian Lectures), Mott (Brain 1892. Vol. 15), angefiihrt von Hugh T. Patrik (Dieses Archiv Bd. XXV. 3. Heft, S. 841 u. f.).

**) Gowers a. a. 0. S. 309. Siehe auch Goldscheider, Diagnostik der Nervenkrankheiten. S. 181.

****) Für die Leitung vom Rückenmark zum Kleinhirn kommen wahrscheinlich noch andere Bahnen in Betracht, namentlich Fibrae arcuatae posteriores und externae; bei ihrer ganz ungewissen Stellung bezüglich der Coordination lässt sich über Störung derselben in dem vorliegenden Falle nichts aussagen. Dagegen kann ich mit Rücksicht auf weiter unten angeführte Ergebnisse der *iteratur einen Einfluss der (hier, wie oben bemerkt, noch ungekreuzten) Pyramidenbahnen resp. deren Läsionen auf die Ataxie nicht ganz yon der Hand weisen, möchte indessen der Kleinhirnseitenstrangbahn eine weit grössere Wichtigkeit zuschreiben. 
(wie die Ataxie, aber später) verschwunden, das Trauma hat demnach diese Bahnen nicht zerstört, sondern nur für einige Zeit leitungsunfähig gemacht. Kehren wir zu unserem Querschnittsbilde zurück und wenden uns nach innen von den zum Corpus restiforme ziehenden Fasern der Kleinhirnseitenstrangbahn, so gelangen wir an die "aufsteigende" Quintuswurzel und deren Kern. Dass beide durch den Process recht sehr gelitten haben, geht schon daraus hervor, dass ein unerträglicher Schmerz im linken Auge und dessen Umgebung ausser dem Schwindel das erste Symptom des Insults war, dass der Corneal- und Conjunctivalreflex links von Anfang an fehlte, dass die Hyperalgesie sich rasch in eine Anaesthesie verwandelte, dass endlich im Gebiete des linken Trigeminus ein Herpes auftrat. Viel schwieriger erscheint mir die Beantwortung der Frage: Können wir die Ausdehnung dieser Anaesthesie zur Höhenbestimmung benutzen? Mit gewissen Einschränkungen glanbe ich, diese Frage bejahen zu dürfen. Die Trigeminusaffection ist auf einen grossen Theil des ersten, einen kleineren des zweiten Astes beschränkt, die Schleimhautzweige des Mundes waren nur vorübergehend an der Störung betheiligt. Am meisten hat der Nerv. nasociliaris gelitten. Pathologische Beobachtungen $*$ ) und experimentelle Untersuchungen $* *)$ machen es sehr wahrscheinlich, dass die Mundschleimhautäste und Geschmacksnerven im proximalsten Theile des aufsteigenden Quintuskerns endigen, dass auch die übrigen Wurzeln des dritten Astes weiter oben in den Kern einstrahlen, als die der beiden ersten, dass endlich "die Zuzüge von den distalsten Partieen der Medulla oblongata (zugleich die ventrale Seite der aufsteigenden Wurzel einnehmend) und des Halsmarks hauptsächlich dem Ramus I gelten" (Bregmann a. a. 0.). Acceptiren wir diese Resultate $\left.{ }^{* * * *}\right)$, so gelangen wir nothwendig zur Annahme einer Affection des untersten und mittleren Theiles der aufsteigenden Wurzel nebst Kern, und zwar würde der ventrale Theil am intensivsten ergriffen sein müssen. Diese Annahme stimmt aber vollkommen überein mit den früher von uns gezogenen Höhengrenzen des Herdes. Ob die stärkere

*) Eisenlohr, Dieses Archiv Bd. XIX. S. 314.

**) E. Bregmann, Ueber experimentelle aufsteigende Degeneration motorischer unp sensibler Hirnnerven. Jahrb. f. Psychiatrie 1892. S. 88 u. f.

***) Anmerkung bei der Correctur: Zur Feststellung der Localisation des Cornealreflexes habe ich an Kaninchen und Katzen die sogenannte ,aufsteigende" Quintuswurzel, vom. Halsmark aufwärts gehend, partiell zerstört. Die Versuche sind noch nicht abgeschlossen, haben jedoch bisher ergeben, dass eine Verletzung der Quintuswurzel resp. des Kerns in der Höhe der Eröffnung des Centralcanals den Cornealreflex derselben Seite bei Kaninchen aufheben kann. 
Läsion des Schmerz- und Temperaturgefübls auf besondere Theile des Kerns hindeutet, lasse ich dahingestellt, um mich nicht in leeren Hypothesen zu verlieren*). Dagegen glaube ich mit grösserem Rechte die yorwiegende Betheiligung jener Sensibilitätscategorien an der rechten Körperhălfte für die Querschnittsbestimmung benutzen zu können. Ich gehe dabei von der (jetzt ziemlich allgemein acceptirten) Ansicht aus, dass die Fasern für Schmerz- und Temperaturempfindung in das Hinterhorn derselben Seite einstrahlen, dass ihre centralen Fortsetzungen sich kreuzen (hauptsächlich in der weissen Commissur), im Vorderseitenstrange der anderen Seite aufwärts steigen, in der Höhe der Pyramidenkreuzung medialwärts rücken und sich weiter oben lateral an die aus der Schleifenkreuzung hervorgehenden Bündel anlegen. Letztere kommt dadurch zu Stande, dass aus den Hinterstrangskernen (zuerst, vom Rückenmark aus gerechnet, aus dem Goll'schen, dann aus dem Burdach'schen Kerne) Fibrae arcuatae internae im Bogen auf die andere Seite und dort im Vereine mit den Vorderseitenstrangresten als Olivenzwischenschicht resp. Schleife cerebralwärts ziehen (Edinger). Da in die Hinterstrangskerne wahrscheinlich Fasern für Tastgefühl, Ortssinn, Drucksinn der gleichen Körperhälfte einstrahlen, so werden wir deren Bahnen auch in dem medialen Theile der gegenüberliegenden Schleifenanlage erwarten müssen, die Anordnung der verschiedenen sensiblen Fasercategorieen der rechten Körperhälfte (bis auf das Gesicht) wird demnach etwa folgende sein: Medial vom aufsteigenden Kerne des linken Trigeminus Bahnen für Schmerz- und Temperaturempfindung, in der von uns angenommenen Höhe noch von sehr wenigen, weiter oben von zahlreicheren Fibrae arcutae internae durchzogen, nach innen von diesen, bis zur Raphe reichend, Fasern für die übrigen Qualitäten. In unserem Falle besteht neben der Trigeminusanästhesie der linken Seite eine starke Beeinträchtigung des Schmerz- und Temperaturgefühls (namentlich für Kälte) auf der rechten Körperhälfte (Analgesie bis zur Schulter, Thermanaesthesie bis zum Unterkieferrande). Ortssinn, Drucksinn, electrocutane Empfindung der rechten Seite haben eine weit geringere Einbusse erlitten, und es beschränkt sich dieselbe auf bestimmte Regionen, besonders Brust und Rücken. Der von uns angenommene Herd

*) Hösel (Dieses Archir Bd. XXIV. 2. Heft, S. 476) stellt wohl den aufsteigenden Quintuskern allein in eine Parallele mit dem Hinterhorn, den sogenannten sensibeln Kern des Trigeminus aber nennt er ein „hauptsächlich dem inneren Burdach'schen Kern entsprechendes Gebilde". Diese Analogie würde sehr wohl mit der relativen Intactheit des Tastsinns in unserem Falle übereinstimmen, 
in der linken Hälfte der Mednlla oblongata muss sich demnach weit medialwärts erstrecken, die Vorderseitenstrangreste in toto treffen, während die Schleifenanlage seine mediale Begrenzung bildet, jedenfalls nur eine geringe Schädigung erlitten hat. Ob ein paar dazwischen verlau* fende Hypoglossuswurzeln oder Kerntheile dieses Nerven mit lädirt sind, lasse ich dahingestellt, denn die Zungenaffection ermöglicht es uns nicht, eine Entscheidung nach dieser oder jener Richtung zu treffen. Etwas günstiger liegt die Sache, wenn es sich darum handelt zu entscheiden, ob Fibrae arcutae internae der linken Seite auf ihrem Wege zur Schleifenkreuzung getroffen sind, wie es a priori als sicher erscheinen muss, besonders wenn wir den Querschnitt etwas weiter cerebralwärts anlegen. Berücksichtigen wir die relativ geringe Zahl dieser Fasern, so würde die unbedeutende Vergrösserung der Tastkreise an der linken Halsseite, Schulter, weniger am Fussrücken auf eine Läsion jener Fibrae arcuatae hindeuten. Eine weitere Trennung vorzunehmen, weil gerade die dem Burdach'schen Kerne entstammenden Bahnen (Schulter) am meisten tangirt sind, halte ich nicht für opportun. Wir gehen daher in unserem Querschnittsbilde weiter dorsalwärts und gelangen somit an die Hinterstrangskerne, den Beginn des „Respirationsbündels" und der Ala cinerea. Für eine Betheiligung dieser Gebilde würde kein Anhaltspunkt vorhanden sein, wenn wir nicht die Insulterscheinungen, besonders die Hyperaesthesie der linken Körperhälfte und die relative Pulsverlangsamung, ausserdem passagere Herdsymptome zu diesem $Z_{w e c k e}$ heranziehen, von denen ich die lange Zeit hindurch bestehende Schlinglähmung, die Reflexhemmung und Sensibilitätsstörung des weichen Gaumens hervorheben will. Wir kommen somit auf Grund der vorhandenen Symptome zu folgendem Resultat: Durch den Insult ist auf der linken Seite der Medulla oblongata ein Areal dicht oberhalb der Pyramidenkreuzung betroffen, welches ventral durch die innere Nebenolive (höher oben durch die Olive), nach aussen durch die Pia mater scharf begrenzt wird; medialwärts ragt es in die linke Schleifenschicht hinein, geht dorsomedial allmälig in normales Gewebe über, erstreckt sich dorsolateral längs des Corpus restiforme bis zum Kleinhirn hinauf. Die grösste Intensität der Läsion (bleibender Herd von schmalelliptischer Form mit der grossen Achse ventrolateral - dorsomedialwärts gerichtet?) erwarten wir innerhalb dieser Zone im ventralen Theile der aufsteigenden Quintuswurzel (nebst Kern) und in der benachbarten Formatio reticularis mit dem Vorderseitenstrangrest, dem motorischen Accessoriusvaguskerne, dessen Wurzel, einzelnen Hypoglossusfädchen; einen weniger hohen Grad von Alteration in der Schleifenschicht, der Kleinhirnseitenstrangbahn und dem Corpus restiforme, am 
wenigsten endlich scheint der Process nach der dorsomedialen Grenze hin eingewirkt zu haben. Für ein Uebergreifen anf die rechte Bulbushälfte spricht von Seiten des Nervensystems kein einziges Symptom. An dieser Stelle mache ich auf die weitgehende Analogie des Krankheitsbildes mit dem Symptomencomplex der Brown-Séquard'schen Halbseitenläsion aufmerksam, sobald man das Trauma aus dem Rückenmark in die Medulla oblongata verlegt. -

Diese ganze Ausführung würde rein hypothetisch sein, wenn sich in der Literatur über diesen Gegenstand nicht einige Fälle auffinden liessen, welche z. Th. eine frappante Aehnlichkeit der klinischen Erscheinungen darboten, und bei denen sich post mortem eine Bulbäraffection in der oben angedeuteten Gegend nachweisen liess. Wenn die quere Ausdehnung der Zerstörung gewöhnlich eine geringere war, als dem von mir umschriebenen Areale entspricht, so stimmt das sehr gut mit meiner Annahme überein, dass sich auch in unserem Falle nur im Innern ein bleibender Herd von relativ geringem Umfange entwickelt hat, und ich verweise bezüglich der Ursache für diese beschränkte Erweichung auf die weiter unten folgende Begründung.

Die beiden für mich werthvollsten Analoga hat Wernicke in seinem "Lehrbuch der Gehirnkrankheiten" ") angeführt:

Senator (Dieses Archiv XI. Band). 56jähriger Mann erwacht eines Morgens mit Schwindel, Unfähigkeit zu gehen, Neigung nach links zu fallen, Sprach- und Schlingstörung, Gefühl von Kälte in der linken Gesichtshälfte.

Status am folgenden Tage: Freies Sensorium, gesteigerte Pulsfrequenz, Neigung nach links zu fallen beim Stehen und Gehen. Im Liegen keine Ataxie, gute Ausführung der Bewegungen. Auch an Zunge und Gaumen keine Lähmung, nur Schluckbeschwerden und Heiserkeit (wie es scheint, unvollständige Schliessung der Stimmbänder), Sensibilität in der linken Gesichtshälfte und auf der ganzen rechten Körperhälfte fast vollständig erloschen. Linke Zungenhälfte, Cornea, Conjunctiva, Nasenhöhle auch anaesthetisch. Hautreflexe normal, Patellarreflex fehlt beiderseits. Gehör, Geschmack normal.

Section: Ein durch Thrombose im Bereiche der linken Vertebralis bedingter Erweichungsherd im äusseren unteren Theile der linken Hälfte des verlängerten Marks. Atheromatöse Entartung der Hirnarterien. Putride Bronchitis. Thrombus der Vertebralis sitzt fest an der Abgangsstelle der A. spinal. anter. und A. cerebellar. inf. post. Letztere blauroth, stark geschlängelt, mit Gerinnsel gefüllt. Die Erweichnng reicht

*) 1. Auflage. II. Bd. S. 224 u. f: 
nach oben nicht bis zum Durchschnitte des Brückenarmes, nach unten nicht ganz bis zur Spitze des Calamus scriptorius, endigt nach innen vor der Raphe und erstreckt sich vielleicht bis zum Vaguskern, nach aussen und seitlich scheint sie weiter zur Brücke hin zu dringen als innen. Pyramide und Olive am frischen Präparat normal, ebenso untere Fläche der Brücke. Nach der Härtung wird constatirt, dass der Herd das linke Corpus restiforme, den angrenzenden Theil des Keilstranges und des Seitenstranges sowie die aufsteigende Quintuswurzel und einen Theil der Vagusfasern durchbrochen hat. Olive, Nebenolive, Hypoglossuswurzeln und -Kern, hiaterer Vaguskern intact. - In diesem Falle ist keine Ataxie constatirt worden, und die Trigeminusanaesthesie bat alle drei Aeste befallen. Beides erklärt sich vielleicht aus der Lage des Erweichungsherdes, welcher etwas weiter cerebralwärts reicht als ich für meinen Fall annehme. Ueber den laryngoskopischen Befund sowie über die Ergebnisse der Temperatursinnprüfung liegen keine Angaben vor.

Duménil (De la paralysie unilatérale du voile du palais. Arch. génér. Avril 1875 obs. 8). 73jähriger Mann. Insult ohne Bewusstseinsstörung. Schlingen unmöglich. Analgesie der ganzen linken Körperhälfte, genau mit der Mittellinie abschneidend; der rechten Gesichtshälfte mit Ausnahme der Gegend des Maxillaris inferior; auch Innenseite der Mundhöhle und der Lippen, Nasenhöhle, Conjunctiva, Cornea in die Anaesthesie mit einbegriffen. Ulcerationen an der rechten Nasenschleimhaut und Oberlippe. Auch Temperaturempfindung an der linken Körperhälfte erloschen, dagegen wird leichte Berührung wahrgenommen. Plantarreflexe erhalten. Grobe Kraft normal. Deutliche Ataxie der rechten Extremitäten. Electrocutane Sensibilität links geringer. Beim Gehen ausser Ataxie auch Gleichgewichtsstörung. Gaumensegel unempfindlich gegen Berührnng, rechte Gaumenbögen unbeweglich, linke normal reagirend. Bei Contraction der linken Gaumenbögen wird Uvula nach rechts gedrängt. Singultus. Besserung des Ganges und der Gaumensegelparese. Stimme hat Falsettimbre.

Section: Ausser Erweichungsherden am rechten Stirn-, Hinterhaupts- und Scheitellappen erbsengrosser Erweichungsherd in der Mitte der Höhe des rechten Corpus restiforme. Umgebung in geringer Ausdehnung verändert, besonders nach aussen und vorn, die Veränderung überschreitet sicher nicht (?) die Breite des Corpus restiforme.

Das von Wernicke beigefügte Fragezeichen erscheint mir wohl berechtigt zu sein, da die aufsteigende Quintuswurzel resp. deren Kern sicher mit in den Bereich des Herdes fällt. Ich halte es ausserdem für sehr wahrscheinlich, dass der Stịmmveränderung eine Stimmband- 
parese zu Grunde liegt. Die dorsale und dem Trigeminusaustritte genäherte Lage der Erweichung würde der Ausdehnung der Quintusaffection auf die Schleimhäute des Mandes und der Lippen wohl entsprechen. Bis auf diese kleine Differenz wiederholen sich in der eben angeführten Beobachtung mit, ich möchte sagen, photographischer Treue fast alle Symptome, welche ich bei meinem Falle beschrieben habe (nur die Zunge scheint intact geblieben zu sein). Besonders hebe ich die isolirte Herabsetzung des Temperatur- und Schmerzgefühls der entgegengesetzten Seite, die Ataxie derselben Seite und die trophischen resp. vasomotorischen Störungen der rechten Gesichtshälfte hervor. Die Sensibilitätsstörung scheint hier dauernd die ganze linke Körperhälfte (mit Einschluss des Gesichts) occupirt zu haben, während bei meinem Patienten die Quintusanästhesie der anderen Seite sehr bald zurückging. Daraus ergiebt sich, meiner lleinung nach, dass auch weiter cerebralwärts gelegene Partieen der linken Bulbushälfte in unserem Falle durch den betr. Process anfangs in Mitleidenschaft gezogen wurden, dass es sich aber um schnell ausgeglichene Störungen handeln muss.

Im folgenden führe ich zwei Fälle an, in denen bei ziemlich gleicher Höhe die Querschnittsausdehnung des Herdes grosse Differenzen zeigt, und wo dementsprechend auch die Symptome variirten.

Senator (Dieses Archiv. Bd. 14. S. 649 u. f.). Analgesie im Bereiche des linken zweiten Quintusastes, Parese der Seitwärtswender der Augen nach links, der rechten Extremitäten, Hypalgesie, Aufhebung des Temperaturgefühls, des Gefühls für Lagerung, für passive Bewegungen in den gelähmten Extremitäten, Abschwächung der Hautreflexe, Erhöhung der Sehnenreflexe in den rechten Extremitäten, gesteigerte Pulsfrequenz, Schlucklähmnng, rechtsseitige Hypoglossus-, linksseitige Facialis-Parese, vasomotorische Störungen der rechten Hand. Laryngoskopie unausführbar, Sensibilität des Larynx anscheinend normal.

Section: Thrombose der linken A. vertebralis bis $2-3$ Millimeter über die untere Ponsgrenze hinaus. Verschmälerung der linken Hälfte des Bodens des 4. Ventrikels. Herd reicht vom Abducenskern fast bis zum unteren Ende des Hypoglossuskerns. Grösste Breite in der Mitte der Olive. Seine Querschnittsausdehnung ist folgende: Oben sind Abducensfasern, der mediale Theil des Corpus restiforme, das motorische Feld und die tiefen Querfasern der Brücke zerstört (Quintus also frei!); in der Gegend der grössten Breite: Eminentia teres, Kern und Wurzeln des Hypoglossus, Schleife, motorisches Feld, medialer Theil der Olive und dorsale Pyramidenschicht, beide Acusticuskerne, Vaguskerne (sensorische und motorische), Vaguswurzel, ascendirende Quintuswurzel, Respirationsbündel, Glossopharyngenswurzeln und medialer Theil des Cor- 
pus restiforme; unten: Theil des Corpus restiforme, aufsteigende Trigeminuswurzel, directe Kleinhirnseitenstrangbahn.

Auffallend ist hier die Beschränkung der Trigeminusanaesthesie auf den zweiten Ast. Ich könnte mir dieselbe am besten erklären, wenn der Herd, welcher den Quintus nur im mittleren und unteren Theile der Erweichung tangirt, sich auf den Kern beschränken würde oder wenigstens nur eine kleine Partie der aufsteigenden Wurzel mit in seinen Bereich zöge. Für die Muskelsinnstörungen nimmt Senator die der Raphe zunächst gelegenen Regionen in Anspruch. Ich glaube, man könnte mit grösserem Rechte auf die Läsion der dorsalen Pyramidenschicht recurriren. Unklar bleibt in diesem Falle, warum die Zerstörung der linken Hypoglossuswurzeln und der directen Kleinhirnseitenstrangbahn völlig symptomlos geblieben ist.

Eisenlohr (Dieses Archiv Bd. 19, S. 314 u. f.). Schlingbeschwerden, complete linksseitige Recurrenslähmung mit Anästhesie der linken Kehlkopfhälfte, Lähmung des linken Gaumensegels, Anästhesie aller Qualitäten der drei Quintusäste links, mit Ausnahme der Mund-, Wangen-Zungenschleimhaut, Nystagmus rotatorius bei Wendung der Augen nach rechts, noch mehr bei Drehung nach links, Erhöhung der Pulsfrequenz. Bronchitis putrida, Pneumonie.

Section: Herd („,bulbärmyelitischer") reicht von der Höhe des ersten Cervicalnerven aufwärts bis zum Niveau der Abducenskerne. Quere Ausdehnung: a) Höhe des I. Cervicalnerven: Tubercul. Roland. sin., Accessorinswurzeln, Basis des Hinterhorns; b) untere Oliven: folgt dem Laufe der Accessoriuswurzeln und tangirt aufsteigende Trigeminuswurzel; c) Rautengrube: Dreieck mit der Basis am 4. Ventrikel, der Spitze nach der einstrahlenden Vaguswurzel gerichtet. Zerstört ist: hinterer Vaguskern, Vaguswurzel, solitäres Bündel, vorderer Vaguskern, Theil der aufsteigenden Quintuswurzel, innerste Abschnitte der Kerne der Seitenstränge. d) Areal der Acusticuskerne: der Herd greift auf die inneren Partien des Strickkörpers über, berührt den Quintus wenig; zerstört ist zum kleinsten Theil der innere Quintuskern.

Das Fehlen von Sensibilitätsstörungen der rechten Körperhälfte, von atactischen Erscheinungen, Zungenparese, die Sensibilitätsanomalie des Larynx, die Erhöhung der Pulsfrequenz, vielleicht auch die putride Bronchitis können auf die dorsale Lage des Herdes am Boden der Rautengrube, die Augenmuskelsymptome auf das Hinaufreichen desselben bis in die Höhe der Abducenskerne zurückgeführt werden. Entsprechend der Intactheit des Quintus in dieser Höhe sind die Sehleimbautäste desselben frei geblieben (siehe oben).

Da ich nicht die Absicht habe, hier eine vollständige Sammlung 
aller einschlägiger Fälle zu unternehmen, beschränke ich mich auf die soeben mitgetheilten Daten und greife auch aus der grossen Zahl von Beobachtungen ohne Sectionsbefund nur folgende heraus:

Str ümpell (Archiv für klin. Medic. Bd. 28, S. 43): Schlinglähmung, rechtsseitige Hypoglossus- und Facialisparese, Nystagmus, Neigung zu Strabismus convergens dext., Analgesie und Thermanästhesie der ganzen linken Körperhälfte mit Einschluss des Gesichts.

Es ist anzunehmen, dass der Herd in diesem Falle in die Brücke hineinragt und nicht so weit lateralwärts reicht, dass der Quintnskern. wesentliche Schädigung erlitten hat.

Schwalbe (Deutsche medic. Wochenschrift 1888, No. 35). Rechtsseitige Facialislähmung, linksseitige Extremitätenparese, Zungenparese, wechselständige Empfindungslähmung, und zwar der rechten Gesichts-, linken Rumpfhälfte, der linken Extremitäten; Gaumensegel und Schlundmuskulatur paretisch; vorübergehende Parese des rechten Stimmbandes, Schwerhörigkeit auf dem linken Ohre, Ataxie der linken Extremitäten.

Auch in diesem Falle haben wir Ursache, eine Ueberschreitung der unteren Ponsgrenze durch den Herd zu vermuthen (Facialisbetheiligung, Ataxie der entgegengesetzten Seite) und eine Betheiligung der Pyramidenfasern anzunehmen.

Die folgenden beiden Fälle habe ich in Gottstein's Lehrbuche („Die Krankheiten des Kehlkopfes") gefunden:

Remak (Berliner Gesellschaft für Psychiatrie und Nervenkrankh. Sitzg. 8. Nov. 1880, Berliner klin. Wochenschr. 1881, No. 21, S. 300). Leichter Schlaganfall ohne Bewusstseinsverlust. Schwäche- und Kältegefühl der linken Extremitäten, Schmerzen und Taubheitsgefühl der rechten Gesichtshälfte. Heftiger Schwindel und Neigung nach rechts zu fallen. Rechtsseitige Trigeminusneuralgie, leichte Hemiparesis sin. mit geringer Betheiligung des linken Mundfacialis; Herabsetzung der Hautsensibilität links bis zur Mittellinie des Körpers; undeutlich reagirende Pupillen bei normalem ophthalmoskopischen Befunde und in: tacten Augenbewegungen; Deglutition und Phonation zuerst normal; später Analgesie der rechten Gesichtshälfte, namentlich an der Stirn und am Bulbus, in geringerem Grade an rechter Mundschleimhant und rechter Zungenhälfte, also rechtsseitige Trigeminus- und relative linksseitige Rumpfanästhesie. Bald darauf neuroparalytische Keratitis. Rechtsseitige Posticuslähmung.

Die Läsion dürfte hier hauptsächlich infrapontine Gebiete tangirt haben, wird sich aber ventralwärts (in der Nähe der rechten Pyramide) etwas weiter nach oben erstrecken.

Gottstein-Biermer (a. a. O. S, 305 der 2. Auflage des Lehr- 
buchs): Potator (56j.) erkrankt plötzlich mit „ziehendem Gefühl im Kehlkopf", taumelt, beim Versuche zu stehen, wie ein Betrunkener. Heiserkeit, intensiver Kopfschmerz in der rechten Schädelhälfte, der nach dem Hinterkopfe ausstrahlt. Parästhesien der linken Extremitäten, Wärmegefühl, übermässige Schweisssecretion. Appetit sehr gesteigert, Durstgefühl weniger vermehrt; häufiges Verschlucken beim Essen.

Status 4 Wochen nach der ersten Erkrankung: Temperatur normal, Puls regelmässig, an der rechten Hand mittelvoll, hart, links viel kleiner, etwas weicher, Sklerose der Arterien. Intelligenz normal.

Subjective Beschwerden:

1. Heftiger bes. Nachts auftretender, rechtsseitiger Kopfschmerz.

2. Brenneñdes Gefühl in der linken Körper- u. rechten Gesichtshälfte.

3. Vermehrtes Hunger- und Durstgefühl.

Objective Symptome:

1. Clonische und tonische Zuckungen im rechten Orbicularis palpebrar:; im Uebrigen bis auf Myosis normaler Augenbefund.

2. Analgesie in der Nachbarschaft des rechten Auges und anf der rechten Wangenschleimhaut.

3. Parese der rechten Gaumenhälfte. Anästhesie des Pharynx.

4. Totale Lähmung des rechten Stimmbandes.

5. Verschlucken beim Schlingen von Flüssigkeiten.

6. Linksseitige Hemianalgesie und Hemithermanästhesie sowie mangelnde elektrocutane Schmerzempfindung am Rumpf und den unteren Extremitäten bei völlig erhaltenem Tastsinn und Lagegefühl.

7. Motilität normal; breitspuriger taumelnder Gang und Neigung nach rechts zu fallen.

8. Fehlende Patellarreflexe.

9. Cremaster- und Bauchreflex links stärker als rechts.

Vergleichen wir diese Erscheinungen mit dem Symptomencomplexe, welchen unser Patient zeigt, so lässt sich bis auf unwesentliche Differenzen eine vollständige Uebereinstimmung constatiren.

Die Intactheit des Tastsinns, der Zunge deutet darauf hin, dass der Herd sich nicht so weit medialwärts erstreckt, während er lateral- und ventralwärts wahrscheinlich die untere Ponsgrenze erreicht (Facialisreizung, Betheiligung der Wangenschleimhant). Ob anfangs eine Ataxie bestand, geht aus dem Berichte nicht hervor. Der a. a. O. erwähnten Annahme einer "thrombotischen Verstopfung der Art. cerebr. poster." kann ich nicht beistimmen.

Aus diesen wenigen von mir angeführten Beobachtungen glaube ich folgern zu dürfen, dass aus der grossen Zahl von Symptomen, welche in diesen Fällen constatirt werden konnten, ein einheitliches Krankheits- 
bild resultirt. Die Sectionsergebnisse beweisen, dass dieses Bild auf Erweichungsherde in den seitlichen Theilen der Medulla oblongata zurückgeführt werden muss, dass die zum Theil erheblichen Differenzen hauptsächlich in der Ausdehnung des Krankheitsprocesses (der Höhe und dem Querschnitte nach) begründet sind. Eine kurze Skizze der wichtigsten Erscheinnngen und Varietäten wird das am besten illustriren können.

Der Herd hat seine proximale Spitze:

unterhalb der Ponsgrenze: oberhalb der Ponsgrenze:

Mehr weniger plötzlicher Beginn ohne Bewusstseinsverlust;

Freibleiben des Olfactorius und Options;

Keine Augenmukelstörung

Trigeminusanästhesio derselben Seite, meist auf den ersten Ast oder die beiden ersten Aeste beschränkt. Anfangs häufig Gesichtsneuralgie;

Facialis frei;

Acusticus stets frei.

Stimmband- und Gaumensegellähmung derselben Seite; Sensibilitätsstörungen des Rachens und Larynx; Schlucklähmung; Anomalieen der Pulsfrequenz.

Zungenparese derselben Seite (kann $/$ Zungenparese derselben oder der anfehlen); deren Seite (kann fehlen.)

(Die in einigen Fällen constatirte Hemiparese der anderen Seite gehört wohl kaum noch in das Krankheitsbild hinein.)

Ataxie der Extremitäten der gleichen Seite (kann fehlen);

Analgesie undThermanästhesie dergegenüberliegenden Körperhälfte ohne Betheiligung des Gesichts;
Ataxie auf der anderen Seite (kann fehlen);

Analgesie und Thermanästhesie der gegenüberliegenden Körperhälfte mit Betheiligung des Gesichts.

Andere Qualitäten der Empfindung zeigen nur unwesentliche Störungen.

Neigung nach der Seite des Herdes zu fallen (kann fehlen).

Abschwächang der Sehnenreflexe namentlich auf der Herdseite (variabel).

Hautreflexe fehlen bald auf der Herdseite, bald auf beiden Seiten.

Trophische and vasomotorische Störungen im Gesichte derselben, am Rumpfe und den Extremitäten der anderen Seite (können fehlen).

Welcher pathologische Process liegt diesem Krankheitsbilde zu Grunde? - Bevor ich in eine Besprechung dieser Frage einzutreten versuche, möchte ich darauf aufmerksam machen, dass ein Symptom, welches mein Patient darbietet, bisher unberücksichtigt geblieben ist: das systolische Geräusch am Processus mastoideus dext., das sich nach hin 
ten und unten, weniger nach vorne und oben fortpflanzt und bei Compression der Carotis nahezu ${ }^{*}$ ) verschwindet. Ich gestehe offen, dass"diese Erscheinung erst $3^{1 / 2}$ Monate nach dem Insult von mir constatirt werden konnte, weil ich früher nicht darauf gefahndet habe, und dass mir durch ihre Anwesenheit das bisher so klare Bild erheblich getrübt zu werden schien. Ich musste als Ursache des Geräusches eine Erweiterung incranieller Arterien vermuthen, und wenn auch seine Verbreitung bis zur hinteren Medianlinie an ein Aneurysma der Vertebralis dextra, resp. der Basilaris denken liess, so sprachen die anderen Erscheinungen (Fortpflanzung des Geräusches nach unten mit gleicher Intensität, Abschwächung durch Compression der Carotis) deutlich genug für die Annahme eines Aneurysma der Carotis interna dextra.

Längere Zeit habe ich mich indessen vergeblich bemüht, einen $\mathrm{Zu}$ sammenhang zwischen dem nervösen Symptomencomplexe und einer derartigen Gefässerweiternng ausfindig zu machen. Wohl ist es vorgekommen, dass bei Störungen, welche auf eine acut entstandene, einseitige Affection des Hirnstammes hindeuteten, gerade auf der entgegengesetzten Seite sich ein Aneurysma fand ${ }^{* *}$ ), aber erstens bestanden rein motorische Ausfallserscheinungen, auch von Seiten der Hirnnerven (mit Ausnahme des Acusticus, der anch gelitten zu haben scheint), die sich allenfalls durch eine Pyramidencompression èrklären lassen, zweitens handelt es sich um Vertebral- resp. Basilaraneurysmen; in anderen Fällen endlich **:) waren stets Erscheinungen dabei, welche entweder eine doppelseitige Läsion vermuthen liessen oder der Aneurysmaseite direct entsprachen. Eine locale Erweiterung der rechten Carotis interna müsste nothwendigerweise sensible und motorische Augennerven der rechten Seite zuerst comprimiren, und wenn etwa durch Gegendruck die linke Hälfte des Hirnstammes afficirt würde, so wäre es unverständlich, warum Facialis, Acusticus, Abducens, Trochlearis und Oculomotorius gänzlich unversehrt bleiben sollten, während der Trigeminus, Glossopharyngeus, Vagus, Accessorius und Hypoglossus dauernde, zum Theil recht schwere Schädigungen erlitten haben. Ich glaube daher nicht fehl zu gehen, wenn ich es für sehr unwahrscheinlich halte, dass zwischen dem oben

*) Anmerlung bei der Correctur: Es ist mir nie gelungen, das Geräusch durch Compression der Carotis communis vollständig zu unterdrücken.

*:) Oppenheim und Siemerling, Die acute Bulbärparalyse und die Pseudobulbärparalyse. Charité-Annalen 12. Bd. 1887; u. a. erwähnt: H. Moe ser, Deutsches Archiv f. klin. Med. Bd. 36. Fall III.

***) Moeser a. a. O. Fall I. und II., Oppenheim und Siemerling a. a. O. (eigener Fall), W. Griesinger, Das Aneurysma der Basilararterie. Archir f. Heilkunde 3. Bd. 1862. 
beschriebenen Symptomencomplexe und einem Aneurysma rechtsseitiger Hirnarterien, insbesondere der rechten Carotis interna, ein Causalnexus besteht.

Eine andere Frage drängt sich indessen noch auf, deren Beantwortung mir zur Zeit noch grosse Schwierigkeiten verursacht: Ist das bestehende Geräusch ein ganz sicheres Zeichen für ein intracranielles Aneurysma?*) Oder könnte es sich dabei um andere Gefässveränderungen bandeln? Wenn ich im Folgenden die Gründe anführe, welche mich zur letzteren Annahme bewogen haben, so betone ich ausdrücklich, dass ich ein Aneurysma mit Sicherheit nicht ausschliessen kann. Soviel ich weiss, bedeutet das Auftreten eines systolischen Geräusches innerhalb des Arteriensystems lediglich die Uebergangsstelle von einem engeren Theile des Gefässrohrs zu einem weiteren. In unserem Falle besteht schon in der Carotis communis dextra eine dentliche Erweiterung gegenüber der linken, und wenn wir diese Asymmetrie mit der bestehenden Herzmuskeldegeneration und den ätiologischen Momenten (Alkohol- und Nicotinintoxication) zusammenhalten, so werden wir kaum eine andere Ursache dafür verantwortlich machen können, als eine Arteriosklerose der Halsgefässe, trotzdem an den Temporales, Radiales, Brachiales und anderen sichtbaren Arterien kein Zeichen für Atheromatose sich auffinden lässt.

Nehmen wir an, die durch Sklerose bewirkte diffuse Erweiterung pflanze sich in die Carotis interna bis zu ihrer intracraniellen Verzweigung hin fort, dehne sich event. bis auf die Basilaris aus, so würde in dem Canalis caroticus eine relativ verengte Stelle gegeben sein, welche der Blutstrom passiren müsste, um von der weiten Carotis cervicalis in die ebenso weite Cerebralis zu gelangen. Damit wäre das systolische Blasen sowie sein Verbreitungsbezirk am einfachsten erklärt. Bei Kindern unter einem Jahre vernimmt man bekanntlich öfters an der grossen Fontanelle, zuweilen auch an anderen Stellen des Schädels bis zu den Processus spinosi der Halswirbel hin, ein systolisches Geräusch, und Jurascz erklärt die Entstehung desselben in ganz derselben Weise, wie ich es soeben angedentet habe. Fraglich ist nur, wie lange der Canalis caroticus dem Anprall der Blutwelle widerstehen kann, ohne selber erweitert zu werden. Da ich über Erfahrungen auf diesem Gebiete nicht verfüge, gehe ich zum letzten Abschnitte dieser Arbeit über: Auf wel-

*) Gowers a. a. 0. Bd. II. S. 533, Gerhardt (die betreffende Arbeit konnte ich nicht auffinden), vergl, auch Quincke', Zi emssen's Handbuch 6. Bu. 2. Auflage. S. 423 u. f. 
cher pathologisch-anatomischen Basis hat sich dieser Symptomencom plex aufgebant?

Die bestehende Arteriosklerose und Fettdegeneration des Herzens könnte nach drei Richtungen hin eine Herderkrankung der Medulla oblongata verursachen: durch Blutung, Thrombose oder Embolie. Gegen eine Blutung spricht die Abwesenheit von Bewusstseinsstörungen, die Besserung der meisten Herdsymptome, abgesehen davon, dass eine intrabulbäre Hämorrhagie fast immer zu raschem Exitus führt. Eine Thrombose erscheint mir in Anbetracht des plötzlichen Beginns sehr unwahrscheinlich. Dagegen würde eine Embolie mit circumscripter Erweichung Erscheinungen und Verlauf in befriedigender Weise erklären. Ob der Embolus dem Herzen, den grossen Gefässen oder den Hirnarterien selber entstammt, lasse ich dahingestellt. Es wird sich sein Entstehungsort kaum feststellen lassen, uns interessirt mehr die Stelle, an welcher er haften blieb. Um diese Frage zu beantworten, ist es nothwendig, die in der Literatur niedergelegten Fälle darauf hin zu untersuchen, welche Gefässgebiete sich bei analogen Befunden an der Erweichung betheiligt haben. Meist finden wir die Vertebrales oder die Basilaris als Sitz des Embolus angegeben, der Verschluss war jedoch nicht immer total. Schon Wernicke vermuthet (a. a. O. Bd. II. S. 227), "dass Erweichungsherde in diesen seitlichen Gebieten der Oblongata stets auf Veränderungen der Art. cerebellar. inf. poster. oder ihrer Aeste zu beziehen sind".

Welche Gründe ihn zu dieser Annahme veranlassen, hat er an jener Stelle nicht weiter besprochen. Ich halte es indessen für nothwendig, eine möglichst genaue Orientirung über die Gebiete der hier in Betracht kommenden Hirnarterien vorausgehen zu lassen und erst nach Festlegung ihrer Grenzen uns die Frage vorzulegen: Welche Erscheinungen müssen eintreten, wenn das betreffende Gefäss durch einem Embolus verstopft wird?

Duret's*) treffliche Arbeiten über die Ernährungsgebiete der Hirnarterien sind so allgemein bekannt, dass ich ganz kurz über seine Resultate betreffs der Vertebrales hinweggehen kann: Die Vertebrales, gewöhnlich von verschiedenem Durchmesser (die linke weiter als die rechte), geben etwa $2 \mathrm{Ctm}$. vor ihrer Vereinigung die Art. cerebell. post. infer. ab, weiter oben medialwärts die Art. spin. anter. Die linke Spinal. anterior, gewöhnlich die stärkere von beiden (rechte fehlt oft), läuft in der vorderen Medianfurche caudalwärts, während die rechte daneben

*) Sur la distribution des artères nourricières du bulbe rachidien. Arch. d. physiologie normale te patholog. Mars 1873. - Rech. anat. sur la circulation de l'encéphale. Arch. d. ph. norm. et path. 1874. 
hinzieht. Der Medulla oblongata führen zwei Arten von Aesten dieser Arterien Blat zu: 1. mediane Arterien, welche der Spinalis anterior entstammen, gehen in der Raphe dorsalwärts, vertheilen sich in den medianen Theilen (Olivenzwischenschicht, hinteres Längsbündel, Hypoglossuskerne) und den anderen Kernen am Boden der Rautengrube. Die Pyramiden werden von den Spinal. anter., öfter jedoch aus den Vertebrales selber gespeist. 2. Wurzelarterien (für Hypoglossus aus der Spinal. ant., weniger aus der Vertralis, für Accessorius unten aus der Cerebellar. infer. post., oben aus der Vertebralis selbst, für Vagus und Glossopharyngeus direct aus der Vertebralis) theilen sich in einen Ram. descendens; der längs der Nervenwurzeln nach aussen geht und einen Ram. ascendens, welcher ebenfalls längs der Wurzelfäden läuft, aber intrabulbär im Bogen dorsal- und medialwärts zieht und die Seitentheile der Medulla oblongata vollständig, die Kerne des Ventrikelbodens aber, in denen sie schliesslich ihr Ende finden, nur zum kleinen Theil versorgen, da die überwiegende Zahl von Capillarschlingen den medianen Arterien angehört. Aeste zu den Corpora restiformia werden besonders von den Cerebellares inf. post. abgegeben. Alle diese Arterien sind Endarterien. In ähnlicher Weise versorgt die Basilaris mit ihren Zweigen die Brücke, Acusticus, Facialis, Abducens, Trigeminus. Diese Resultate Duret's kann ich in einigen scheinbar unwesentlichen Punkten nicht bestätigen, möchte aber hier nur auf die Beschreibung näher eingehen, welche er von den Cerebellar. inf: poster. giebt. Er sagt: „Les cérébelleuses inférieures, qui sont situées d'abord à la face antérieure du bulbe, décrivent une courbe qui embrasse les parties latérales de celui-ci et passent à la face postérieure, à peu près deux centimètres au dessus du bec du calamus. Généralement elles décrivent une seconde courbure plus large, qui les porte, en dehors du bulbe, jusque sur le lobule du pneumogastrique; elles se rapprochent enfin une seconde fois de le ligne médiane, mais reposent alors sur le cervelet" - - - (hier folgt eine Schilderung der A. spin. post., welche nach ihrem Abgange von den Cerebellar. post. inf. spinalwärts ziehen, Seitentheile und zum Theile den Boden der Rautengrube versorgen, und der Endverzweigung der Cerebellares: 1. Ast für den Wurm, 2. für Grenze von Wurm und Hemiphäre, 3. für die Hemisphäre). „Les artères cérébelleuses s'anastomosent entre elles à la surface du cervelet; mais ces anastomoses sont rares, elles n'ont pas lieu par un plexus, ni par les plus fines artérioles, mais seulement par des artères d'un quart de millimètre. Lorsqu'on pousse une injection colorée dans une des cérébelleuses, elle revient par l'autre dans le tronc basilaire, q'elle distend, et remplit alors le système des deux côtés". - $\ldots$ 
Im Folgenden will ich versuchen, die Resultate eigener Untersuchungen über den Verlauf und die Verästelnng der Cerebell. inf. post. mitzutheilen, wie ich sie an sieben theils natürlich, theils künstlich injicirten Gehirnen erhalten habe. Ich verdanke das Material der Freundlichkeit des Oberarztes an der inneren Station des Danziger Stadtlazareths, Herrn Sanitätsrath Dr. Freymuth, dem ich an dieser Stelle meinen herzlichsten Dank ausspreche. Gerade die geringfügigen Abweichungen von den Angaben Duret's scheinen mir für den vorliegenden Fall wichtig genug zu sein, um sie hier herzorzuheben.

Wenn anch gewöhnlich von beiden Vertebrales eine Cerebell. inf. post. abgegeben wird, so scheint es doch nicht selten zu sein, dass nur auf einer Seite, häufiger der linken, eine Arterie sich abzweigt, welche durch Caliber und Verlauf resp. Verästelung als untere Kleinhirnarterie sich kennzeichnet. Auf der anderen Seite vertreten dann mehrere kleinere $(1 / 4-1 / 2 \mathrm{Mm}$. dicke) von der Vertebralis kommende Aestehen die Stelle der fehlenden Cerebellaris, aber nur für die Medulla oblongata, während die Kleinhirnzweige durch eine stärker ausgebildete Art. cerebell. inf. anterior ersetzt werden. Die normal entwickelte Cerebell. inf. post. geht meist in der Weise von der lateralen Wand de: Vertebralis $a b$, dass sie mit dem caudalen Ende derselben einen spitzen Winkel bildet, seltener mit dem proximalen Abschnitte; im rechten Winkel sah ich sie niemals abgehen. Die Theilungsstelle liegt $12-20 \mathrm{Mm}$. nnterhalb der Vereinigung der Vertebrales, an der Austrittsstelle von Hypoglossuswurzeln (je nach der Höhe des Abgangs unteren oder mittleren Wurzelfäden). Ein Querschnitt durch die Oblongata an diesem Punkte zeigt eben vollendete Pyramidenkreuzung oder Ausbildung der inneren Nebenolive oder anch den Beginn der Olive selbst. Mebrmals habe ich (im Gegensatze zu Duret) beobachtet, dass die Cerebellaris dicht am Ursprunge ein par winzige Zweige zu den Hypoglossusfäden sendet. Sie schlingt sich dann um den lateralen Theil des Bulbus herum, und zwar beschreibt sie entweder (in der Mehrzahl der untersuchten Präparate) 3-8 Mm. unterhalb des caudalen Poles der Eminentia olivaris einen nach oben hin concaven Bogen und biegt dann parallel mit dem lateralen Olivenrande cerebralwärts $\mathrm{nm}$, oder sie geht quer über die Olive hinweg (ungefähr $3 \mathrm{Mm}$. oberhalb des unteren Poles). Auf dieser ca. $10 \mathrm{Mm}$. langen Strecke giebt die Arterie mehrere (2-5) Aeste ab. Die stärkeren und zugleich längeren (4-6 Mm. vom Ursprunge sich abzweigend) gehen zuerst eine Strecke parallel mit den Wurzelfasern des bulbären Accessoriusabschnitts ${ }^{*}$ ) oder des unteren resp. mittleren Vagus (in

*) Duret giebt an, dass nur der spinale Accessorius von der Cerebellaris versorgt wird. Diesen Befund habe ich nicht bestätigen können. 
einem Falle auch mit den an der Grenze von Vagus und Glossepharyngeus laufenden Fäden) and theilen sich dann in kleinere Zweige, welche die ursprüngliche Richtung innehaltend, mit den Wurzelfasern nach der Peripherie hinziehen, und in stärkere, welche dorsomedialwärts in spitzem Winkel abbiegen und, den Nervenfädchen dicht angelagert, mit ihnen in den Bulbus dringen. Im Innern beschreiben sie einen flachen, dorsomedialwärts gerichteten Bogen und gelangen nach Abgabe von Aesten an den Trigeminuskern und-Wurzel, sowie an die dem Kerne medial anliegenden Theile der Formatio reticularis zu den grauen Massen am Boden der Rautengrube. Hier communicirt ihr Capillarnetz mit dem. jenigen medianer Arterien (aus der Spinalis anterior und Vertebralis), Neben diesen Wurzelarterien habe ich stets auch kleinere Aeste gesehen, welche zwar 1-2 Mm. peripher von jenen die Cerebellaris verliessen, am lateralen Olivenrande jedoch umbogen und hier in die Oblongata einstrahlten, ohne mit Wurzelfasern in Berührung zu kommen. Innerhalb des Bulbus laufen sie zuerst parallel (ventral) zu den oben beschriebenen Wurzelästen, trennen sich aber unter fortdauernder Abgabe von Zweigen an die Oliven und deren Nachbarschaft, indem sie die rein mediale Richtung beibehalten, und verlieren sich in der grauen Substanz zwischen Olive und Quintuskern*).

Nach Abgabe dieser Aeste dringt die Cerebellaris zwischen Vagus und Accessorius resp. zwischen Vaguswurzeln nach hinten und gelangt (je nach der Höhe ihres Ursprungsortes) dicht unterhalb oder bis zu $6 \mathrm{Mm}$. oberhalb des Obex in die Nähe der hinteren Medianfurche. Ausser kleinsten, sehr inconstanten Zweigen für die Hinterstrangskerne, etwas stärkeren für das Corpus restiforme giebt sie auf dieser Strecke noch eine Spinalis posterior ab, ein sebr unansehnliches, im Sulcus dorsalis lateralis abwärts laufendes Gefäss, welches sebr bald durch direct aus der Vertebralis entspringende Collateralen verstärk $\hat{t}$ wird. Von da ab liegt die Cerebellaris in einer Furche des Unterwurms und

*) Einen analogen Verlauf nehmen die von der Vertebralis selber abgehenden Wurzelarterien und deren Begleiter. An frontalen, namentlich aber an sagittalen Schnittserien durch den Hirnstamm konnte ich ron dem Ende der Pyramidenkreuzung bis zur Brücke mir ihren intrabulbären Weg zur Anschauung bringen. Sie folgen sich in regelmässigen Zwischenräumen von $0,6-0,8 \mathrm{Mm}$., in einer Stärke ron 150-200 $\mu$. Thre Querschnitte (an sagittalen Serien) bilden bei ihrem Eintritte in die Oblongata zwei oder drei sanft dorsalwärts ansteigende, nahezu parallele Linien. Weiter medianwärts entfernen sie sich von einander (siehe oben). Die medianen Arterien besitzen gewöhnlich ein grösseres Lumen (bis zu $300 \mu$ ) und ziehen in Abständen von ca. $1 \mathrm{Mm}$. schräge von der ventrocaudalen Fläohe dorsal- und zugleich proximalwärts. 
steht nur durch kleine Aeste, hauptsächlich für den Strickkörper, mit dem Bulbus in Verbindung. Sie wendet sich bei dem gewöhnlichen tiefen Ursprunge in enger Curve lateral- und aufwärts, am lateralen Rande des Tubercul. cuneatum angelangt, schwenkt sie in flachem Bogen wieder medianwärts, folgt dabei ungefähr der Diagonale des Corpus restiforme, dem sie zahlreiche Zweige sendet, und theilt sich endlich wenige Millimeter unterhalb der Striae acusticae in mehrere, gewöhnlich drei Aeste, von denen einer unter der Pyramide des Wurms die Anastomose mit der gleichnamigen Arterie der anderen Seite vermittelt, der zweite an der Grenze von Wurm und Hemisphäre läuft, der dritte endlich sich in den hinteren unteren Partien der Hemisphäre verästelt. In den Plexus chorioideus medialis des 4. Ventrikels dringen mehrere Zweige der Cerebellaris gewöhnlich vom Scheitel der medialen Curve her. Bei hohem Ursprunge gehen dieselben schon ab, bevor die Medianlinie erreicht ist. Die Arterie biegt dann ein wenig lateralwärts nach oben um, sendet noch einige grössere Zweige für den Strickkörper und löst sich, ohne die dritte Curve za beschreiben, in ihre Endäste auf. Die Striae acusticae scheinen mir stets von der Basilaris aus versorgt zu werden. - Ich habe eine Canüle von der Vertebralis aus bis an die Abgangsstelle der Cerebellaris inf. post. herangebracht, dicht oberhalb derselben eine Ligatur um die Vertebralis gelegt, ebenso um die Bifurcationsstellen der Basilaris am caudalen und proximalen Brückenrande, um alle übrigen Kleinhirnarterien - kurz ich habe alle Hirngefässe mit Ausnahme einer Cerebellaris inf. post. von der Injection ausgeschlossen. Trotzdem wurden nicht nur alle 3 Kleinhirnarterien beider Seiten voll injicirt, sondern es füllten sich allmählich auch die Vertebrales, die Basilaris mit ihren Aesten bis zu den Ligaturstellen strotzend an (siehe Duret a. a. 0.).

Diese Ergebnisse bilden die Basis für meinen Versuch, den Symptomencomplex in unserem Falle ausreichend zu erklären. Nehmen wir einen embolischen Verschluss der linken Cerebellaris infer. poster. an dem Orte ihres Abganges von der Vertebralis an (event. durch einen auf dem Theilungswinkel reitenden Embolus), denken wir uns die Verlegung des Gefässrohrs auf weuige Millimeter ausgedehnt: was wird die Folge sein?*) Zuerst Aufhebung der Circulation in dem lateralen und dorsalen Abschnitte der linken Oblongatahälfte; für den weiteren Ver-

*) Dass eine Erweiterung der rechten Vertebralis, ohne die rechte Bulbushälfte erheblich zu comprimiren, dureh Gegendruck einen acuten Verschluss der linken Cerebelliris verursachen könnte, müchte ich stark bezweifeln. 
lauf und die anatomischen Folgen dieser Absperrung der Blutzufuhr wird es notbwendig sein, drei Gebiete auseinander zu balten:

1. Das dorsomediale, dem Boden der Rautengrube benachbarte Gebiet wird, wie wir gesehen haben, hauptsächlich von den medianen Arterien und den Spinales posteriores versorgt. Da aus der Vertebralis kommende Collateralen das Lumen der cerebellaren Spinalis posterior erheblich übertreffen, die von Wurzelarterien gespeisten Capillarschlingen der grauen Kerne von den Medianen her sich mühelos mit arteriellem Blute injiciren werden, so kann sich die entstandene Circulationsstörung hier sofort wieder ausgleichen und lediglich zu schnell vorübergehenden Insulterscheinungen Anlass geben: Hyperästhesie der linken Körperhälfte, Pulsverlangsamung, Anästhesie des Rachens und des Ganmens. Auch die dem verschlossenen Gefässgebiete benachbarten Theile werden für kurze Zeit in der Füllnng ihrer Blutbahnen anomale Verhältnisse darbieten, darum können wir auch die Betheiligung der rechten Gesichtshälfte, die anfängliche Ausbreitung der Sensibilitätsstörung auf alle Trigeminusäste der linken Seite, endlich vielleicht die Aufhebung des rechten Patellarreflexes dem Bilde der Insultsymptome einfügen. Die längere Zeit nachweisbare Schlinglähmung, bei welcher neben sensiblen auch motorische Störungen mitspielen, bildet den Uebergang zu der zweiten Gruppe von Erscheinungen.

2. Etwas ungünstiger gestaltet sich die Herstellung einer normalen Circulation in dem dorsolateralen Gebiete des verlängerten Markes (Corpus restiforme, directe Cerebellarbahn, ganz unten event. noch ungekreuzte Pyramidenbündel) und dem Kleinhirn. Hier müssen erst die bestehenden Anastomosen zwischen den Endästen der. Kleinhirnarterien derart erweitert werden, dass der Blutstrom rückwärts in die Cerebell. post. inf. mit genügender Stärke bis an den Sitz des Embolus gelangen kann: Mehr oder weniger passagere Herdsymptome, von denen ich die Neigung nach links zu fallen, die Ataxie der linken Extremitäten, die Aufhebung des linken Patellarreflexes besonders hervorheben möchte ${ }^{*}$ ).

3. Aus der vom Embolus verlegten Anfangsstrecke der Cerebellaris gehen bei dem gewöhnlichen tiefen Ursprunge derselben neben wenigen Hypoglossuszweigen hauptsächlich Arterien hervor, welche obere Accessorius- und untere Vaguswurzeln, deren motorischen Kern, aufsteigende Quintuswurzeln nebst Kern (vorwiegend ventrale Theile desselben), endlich die dem Quintuskerne medial anliegenden Gebilde bis zur Schlei-

*) Auf das Verhalten der Hautreflexe gehe ich wegen der Unsicherheit, welche heute noch über ihre Veränderungen bei bulbären Affectionen herrscht, nicht näher ęin, 
fenkreuzung hin mit Blat versorgen. Auch die innere Nebenolive resp. der unterste Theil der Olive wird mitgerechnet werden müssen, wenn die für sie bestimmten Zweige auch weiter peripherwärts sich von der Cerebellaris abzulösen pflegen. Soweit nicht mediane und andere von der Vertebralis kommende Arterien die Ernährung dieser Theile übernehmen, müssen letztere necrotisch werden. Es wird sich demnach ein kleiner Erweichungsherd innerhalb dieser Zone etabliren, dessen Umgebung allmälig in normales Gewebe übergeht. Diesem Herde entsprechen die dauernden Symptome, besonders wenn sie mit trophischen Störungen vergesellschaftet wird: Anästhesie im ersten und zweiten Aste des linken Trigeminus mit Herpeseruption, Analgesie und Thermanästhesie der rechten Seite, ebenfalls mit Herpes, geringe linksseitige Hyoglossus- und Gaumenparese, nahezu totale Stimmbandlähmung (mit consecutiver Atrophie?*)).

An einen (meist embolischen) Verschluss der Art. cerebellar. infer. poster. werden wir in jedem Falle denken müssen, in welchem von den beiden einander gegenüber gestellten Krankheitsbildern (siehe oben) dasjenige vorliegt, bei welchem wir eine unterhalb der caudalen Ponsgrenze endigende Läsion anzunehmen berechtigt waren. Die kleinen Differenzen erklären sich genügend aus den Varietäten des Ursprungs und Verlaufs der Cerebell. inf. post. Sprechen die Erscheinungen dagegen für einen in die untere Ponshälfte hineingehenden Herd, so werden wir den Sitz des Embolus in die letzte Strecke der Vertebralis vor ihrer Confluenz mit der anderen verlegen. Die variable Abgangsstelle der Spinalis anterior gäbe dann einen ausreichenden Grund auch für das Fehlen resp. Vorhandensein von Hemiparesen mit Einschluss des Hypoglossus ab.

In wie weit solche theoretisch begründeten Annahmen ihre Berechtigung haben, kann erst in der Zukunft durch eine sorgfältige Sammlung klinischer und anatomischer Beobachtungen entschieden werden. Einen klinischen Beitrag zu liefern, war der Zweck dieser Arbeit.

Danzig, 21. März 1894.

*) Anmerkung bei der Correctur: Bis zum März 1895 ist die Recurrenslähmung erheblich zurückgegangen; es besteht jetzt nur eine Parese und Verschmälerung des linken Stimmbandes. 